\title{
Genetic modification increases the survival and the neuroregenerative properties of transplanted neural stem cells
}

\author{
Irina Korshunova, ${ }^{1}$ Sina Rhein, ${ }^{2}$ Diego García-González, ${ }^{1}$ Ines Stölting, ${ }^{2}$ Ulrich Pfisterer, ${ }^{1}$ \\ Anna Barta, ${ }^{1}$ Oksana Dmytriyeva, ${ }^{3,4}$ Agnete Kirkeby, ${ }^{5,6}$ Markus Schwaninger, ${ }^{2}$ \\ and Konstantin Khodosevich ${ }^{1}$ \\ ${ }^{1}$ Biotech Research \& Innovation Centre, University of Copenhagen, Copenhagen, Denmark. ${ }^{2}$ Institute for Experimental \\ and Clinical Pharmacology and Toxicology, University of Lübeck, Lübeck, Germany. ${ }^{3}$ Department of Biomedical Sciences, \\ ${ }^{4}$ Novo Nordisk Foundation Center for Basic Metabolic Research, and ${ }^{5}$ Department of Neuroscience, Faculty of Health and \\ Medical Sciences, University of Copenhagen, Copenhagen, Denmark. ${ }^{6}$ Department of Experimental Medical Science and \\ Wallenberg Centre for Molecular Medicine, Lund University, Lund, Sweden.
}

Cell therapy raises hopes high for better treatment of brain disorders. However, the majority of transplanted cells often die soon after transplantation, and those that survive initially continue to die in the subacute phase, diminishing the impact of transplantations. In this study, we genetically modified transplanted human neural stem cells (hNSCs), from 2 distant embryonic stem cell lines (H9 and RC17), to express 1 of 4 prosurvival factors - Hif1a, Akt1, Bcl-2, or Bcl-xl - and studied how these modifications improve short- and long-term survival of transplanted hNSCs. All genetic modifications dramatically increased survival of the transplanted hNSCs. Importantly, 3 out of 4 modifications also enhanced the exit of hNSCs from the cell cycle, thus avoiding aberrant growth of the transplants. Bcl-xl expression provided the strongest protection of transplanted cells, reducing both immediate and delayed cell death, and stimulated hNSC differentiation toward neuronal and oligodendroglial lineages. By designing hNSCs with drug-controlled expression of Bcl-xl, we demonstrated that short-term expression of a prosurvival factor can ensure the long-term survival of transplanted cells. Importantly, transplantation of Bcl-xl-expressing hNSCs into mice suffering from stroke improved behavioral outcome and recovery of motor activity in mice.

Authorship note: IK and SR contributed equally to this work. MS and KK contributed equally to this work.

Conflict of interest: The authors have declared that no conflict of interest exists.

Copyright: ( 2020, American Society for Clinical Investigation.

Submitted: November 16, 2018 Accepted: January 22, 2020 Published: January 30, 2020.

Reference information: /CI Insight. 2020;5(4):e126268.

https://doi.org/10.1172/jci. insight.126268.

\section{Introduction}

Cell therapy represents a promising approach to treating brain diseases. Neural stem cells (NSCs) or precursor cells are usually transplanted at the site of injury to restore function in damaged brain tissue. Although such transplantations have provided some positive effects in several animal models of brain diseases, they are also accompanied by a high death rate of transplanted cells (reviewed in refs. 1, 2). The debris from dead cells not only stimulates inflammation around transplants, but also and more importantly, eventually negates the positive effect of transplantations. Two distinct phases of cell death are characteristic for predifferentiated transplanted cells: immediate cell death within the first week after transplantation and continuous cell death over the following time period. Thus, the bulk of transplanted cells often die during the first phase, and the majority of initially surviving cells die progressively during the following weeks or months (3-6). In other cases, when undifferentiated or partially differentiated stem cells of embryonic or pluripotent stem cell origin are transplanted, it is difficult to evaluate cell survival because of the high proliferation rate of transplanted cells. However, uncontrolled proliferation of transplanted cells is undesirable in cell therapy because it might result in the formation of brain tumors $(7,8)$. Thus, it is important to increase survival of transplanted cells without affecting their proliferation.

Survival of transplanted cells depends both on extrinsic prosurvival signals from surrounding cells and intrinsic survival/death signaling. Attempts have been made to increase the survival of transplanted cells by extrinsic stimulation using growth factors that are secreted by transplanted cells themselves. Although some improvement in survival was observed, growth factor-secreting transplanted cells continued to die, and over time cell death rates became similar to those of unmodified transplanted cells $(9,10)$. 
This can be explained by a loss of growth factor receptors on transplanted cells. Otherwise, signaling from 1 growth factor might not be sufficient to stop cell death, and intracellular apoptotic programs eventually override prosurvival signals. Furthermore, the dependence of transplanted cell survival on extrinsic signals from the local microenvironment also renders these cells vulnerable to cell degeneration induced by prodeath factors released from the injured area, and transplanted cells could also acquire the phenotype of surrounding diseased cells (11).

Stimulating cell-autonomous prosurvival programs that do not rely on extracellular signaling represents another means of enhancing transplanted cell survival. Indeed, activating intracellular signaling molecules involved in cell survival should efficiently inhibit cell death because such molecules act downstream of extracellular signals in prosurvival cascades.

In this study, we selected 4 prosurvival molecules that were previously shown to protect neurons during developmental cell death (12), Hif1a, Akt1, Bcl2, and Bcl-xl, and expressed each of their genes in predifferentiated human NSCs (H9 and RC17 human NSCs, hNSCs) that were transplanted into mouse brains. For all 4 types of genetically modified hNSCs, survival was significantly enhanced in comparison with control cells, reaching up to approximately 141-fold higher levels for RC17-derived hNSCs. Importantly, for most genetic modifications, numbers of cells that did not exit the cell cycle 1 month after transplantation were only negligible, and thus there was no uncontrolled growth of the transplants. To identify the period of transgene expression that is required for prosurvival effects, we transduced hNSCs with a lentivirus in which transgene expression could be activated by doxycycline (Dox). We showed that expression of a prosurvival factor for only 1 week after transplantation was sufficient for long-term cell survival in the transplant. Finally, we confirmed that these transplants can be applied to treat disease in a mouse model of stroke, demonstrating that genetically modified hNSCs improve neurological deficit in brain disease.

\section{Results}

Genetic modification of $\mathrm{H} 9$ hNSCs enhances their survival. To analyze survival of transplanted cells, we used human H9-derived NSCs (later H9 hNSCs) that were infected with either control lentivirus or a lentivirus expressing a prosurvival gene (Figure 1A). This cell type for transplantation was chosen because (a) such neural stem/precursor cells are used quite frequently in brain transplantation studies and (b) these cells have the potential to differentiate into neuronal or glial cells, 2 main cell types that are damaged in brain injuries. We confirmed the differentiation potential of these cells by differentiating them into neurons or glial cells in vitro (Figure 1B and data not shown, respectively). To stimulate cell-autonomous survival of transplanted cells, we selected 4 genes that are involved in regulating neuronal survival during prenatal and/or postnatal neurogenesis (13-18), namely, Akt1, Hifla, Bcl2, and Bcl2l1 (Bcl-xl protein). We used myrAkt1 (19), the constitutively active version of Akt1, to strongly stimulate Akt1 signaling. We then subcloned the open reading frames (ORFs) of the prosurvival genes in the lentiviral vector pCDH-CMV-MCS-T2A-EGFP and transduced cultured $\mathrm{H} 9 \mathrm{hNSC}$ by 1 of these viruses.

Because our aim was to evaluate how genetic modification of hNSCs affects transplanted cell survival, it was essential that all transplanted cells express the transgene. When transducing millions of hNSCs in adherent cultures, it is difficult to reach an efficiency of cell transduction above $99 \%$ for 1 lentivirus and even more difficult to attain a cotransduction efficiency above $99 \%$ when using 2 or more lentiviruses. This is mainly due to the dilution of viral vector in the culture medium during transduction. To overcome this problem, we improved the protocol by transducing cells during the culture splitting, subsequently referred to as "split transduction," thereby allowing us to transduce up to several millions of cells at an efficiency of more than $99 \%$ in a short time (see Methods). We implemented split transduction to infect H9 hNSCs either with empty control pCDH-CMV-MCS-T2A-EGFP lentivirus or a lentivirus expressing 1 of the prosurvival ORFs, pCDH-CMV-ORF-T2A-EGFP H9 hNSCs. Importantly, 4 days after transduction, we did not observe any noninfected cells, indicating a complete transduction of the transplanted cells (Figure 1C).

After transduction, hNSCs were cultured for 4 more days without growth factors and were transplanted into the striatum of immunodeficient NOD/SCID- $\gamma$ (NSG) mice. To directly compare survival between control and genetically modified hNSCs, control cells were transplanted into the left and genetically modified cells into the right striatum (Figure 1A). By 1 week after transplantation, only approximately $7 \%$ of control cells had survived, and by 1 month, survival was decreased to approximately $5 \%$ (Figure 1, D and E). Conversely, expression of prosurvival genes dramatically augmented survival of transplanted cells, with an up to 17-fold increase at 3 months after transplantation (Figure 1, D and E). 
A

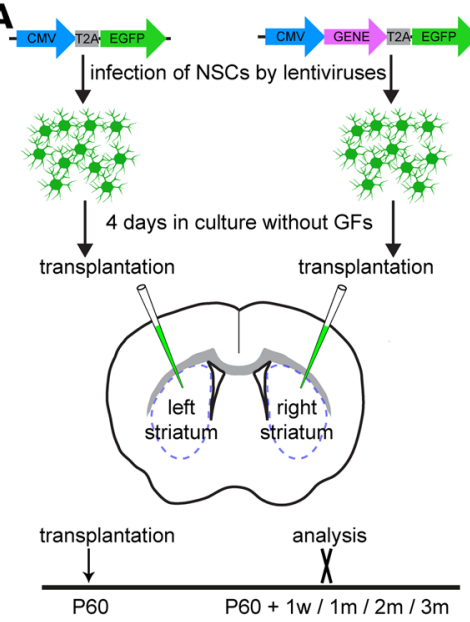

C

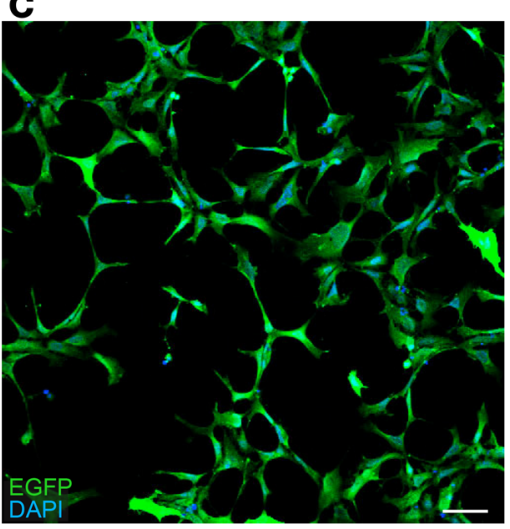

B
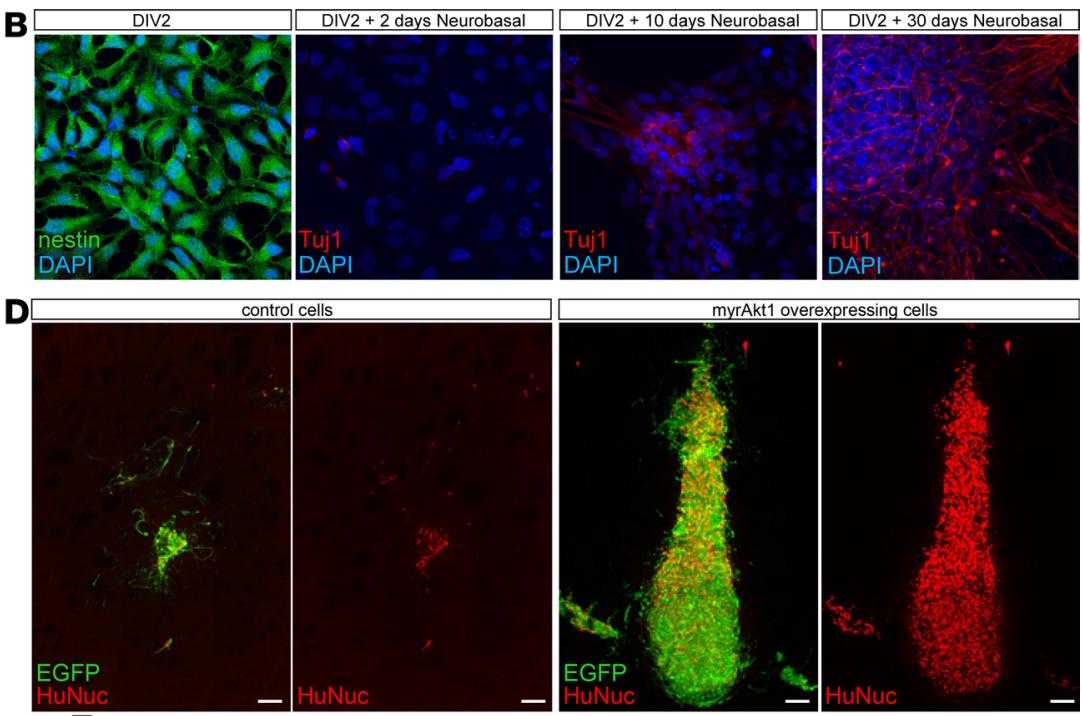

myrakt1 overexpressing cells
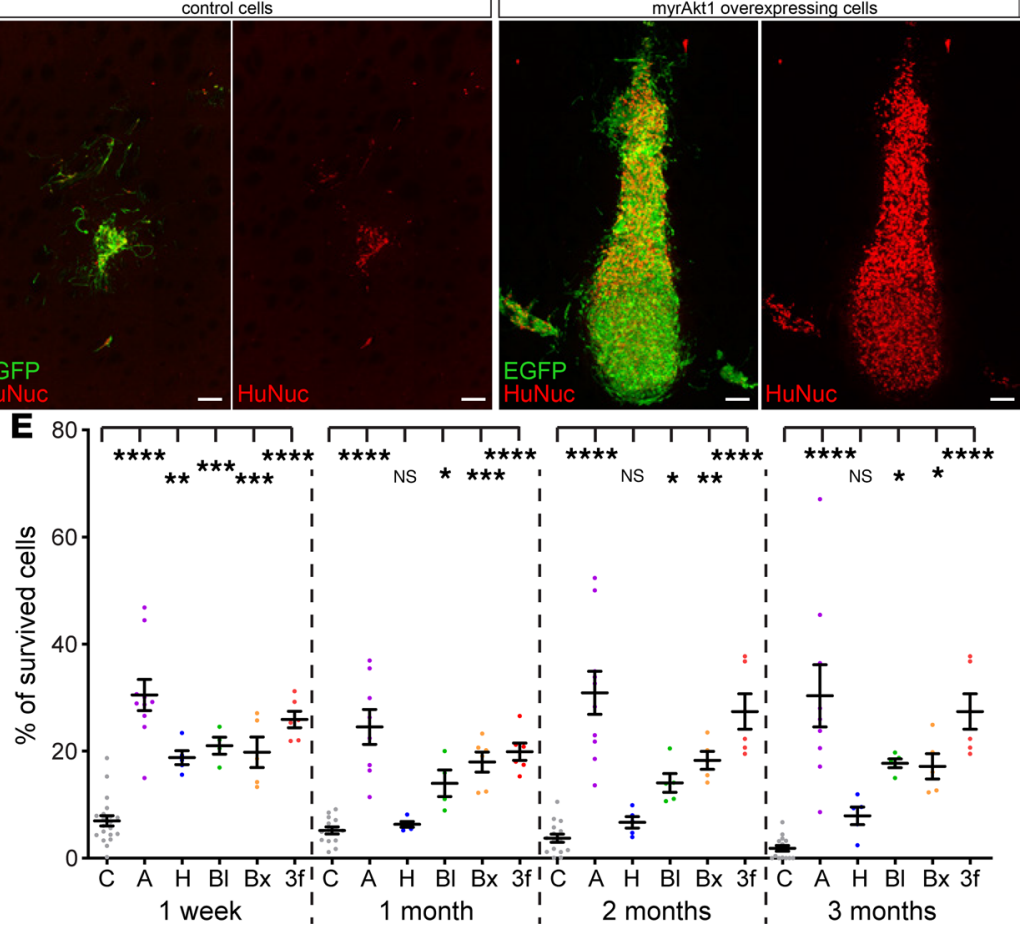

Figure 1. Genetic modification of $\mathbf{H 9}$ hNSCs strongly enhances their survival after transplantation into the striatum. (A) Cultured H9 hNSCs were infected by pCDH-CMV-MCS-T2A-EGFP lentivirus, empty or expressing Akt1, Hif1a, Bc/2, or Bc/2/1 genes. Cells were incubated 4 days in the medium without growth factors and then transplanted into the striatum of 60-day-old NOD/SCID- $\gamma$ (NSG) mice: control cells into the left and genetically modified cells into the right striatum, respectively. Transplants were analyzed 1 week and 1, 2, and 3 months posttransplantation. (B) Differentiation of H9 hNSCs into neurons in vitro. Nestin (neuronal stem cell/precursor marker) and Tuj1 (neuronal marker) staining of H9 hNSCs at different time points of cell culture: day in vitro 2 (DIV2) without neurobasal medium and DIV2 $+2,+10$, and +30 days in the presence of neurobasal medium. (C) H9 hNSCs infected by pCDHCMV-MCS-T2A-EGFP lentivirus, 4 days after infection. (D) Control and myrAkt1-overexpressing H9 hNSCs 1 month after transplantation. (E) Estimation of H9 hNSCs' survival (percentage of total transplanted cells): 1 week and 1, 2, and 3 months after transplantation ( $n=14-20$ controls; $n=5-10$ genetically modified for each time point). C, empty vector; A, Akt1; H, Hif1a; BI, Bcl2; Bx, Bcl2/1; 3f, Akt1 + Bcl2 + Bcl2/1-expressing cells. Mean \pm SEM, 1-way ANOVA with Holm-Šídák post hoc tests. ${ }^{*} P<0.05 ;{ }^{* *} P<0.01 ;{ }^{* *} P<0.001 ;{ }^{* * *} P<0.0001$. Scale bars: $50 \mu \mathrm{m}$ (C), $100 \mu \mathrm{m}$ (D).

As mentioned above, there are 2 distinct phases of cell death in the population of transplanted cells, immediate and continuous cell death. In our experiments, the vast majority of control transplanted cells $(>93 \%)$ died within the first week after transplantation (Figure 1E). Expression of myrAkt1 was most efficient, resulting in $30 \%$ survival of transplanted cells. Three other prosurvival genes, Hifla, Bcl2, and $B c 1211$, protected transplanted cells to a similar extent; namely, $20 \%$ of cells survived (Figure 1E and Supplemental Figure 1; supplemental material available online with this article; https://doi.org/10.1172/jci. insight.126268DS1). Control cells continued to die and their numbers decreased to $1.8 \%$ of initially transplanted cells at 3 months after transplantation. Importantly, $A k t 1, B c l 2$, and $B c l 2 l 1$ not only significantly protected transplanted cells from immediate death but also abrogated continuous cell death (Figure 1E and Supplemental Figure 1). After 1 week, the population of surviving cells expressing $A k t 1, B c l 2$, or $B c l 2 l 1$ was stable, although $A k t 1$ - and $B c l 2$-expressing transplants exhibited a small decrease in cell survival between 1 week and 1 month after transplantation. Interestingly, at 1 month after transplantation, the number of surviving Hifla-expressing cells dropped from 20\% to $6.3 \%$ (Figure 1E), whereas from 1 month after transplantation onward, cell death in these transplants was inhibited. Thus, it is likely that Hifla expression only delayed immediate cell death but abolished continuous cell death. The prosurvival effect by genetic 

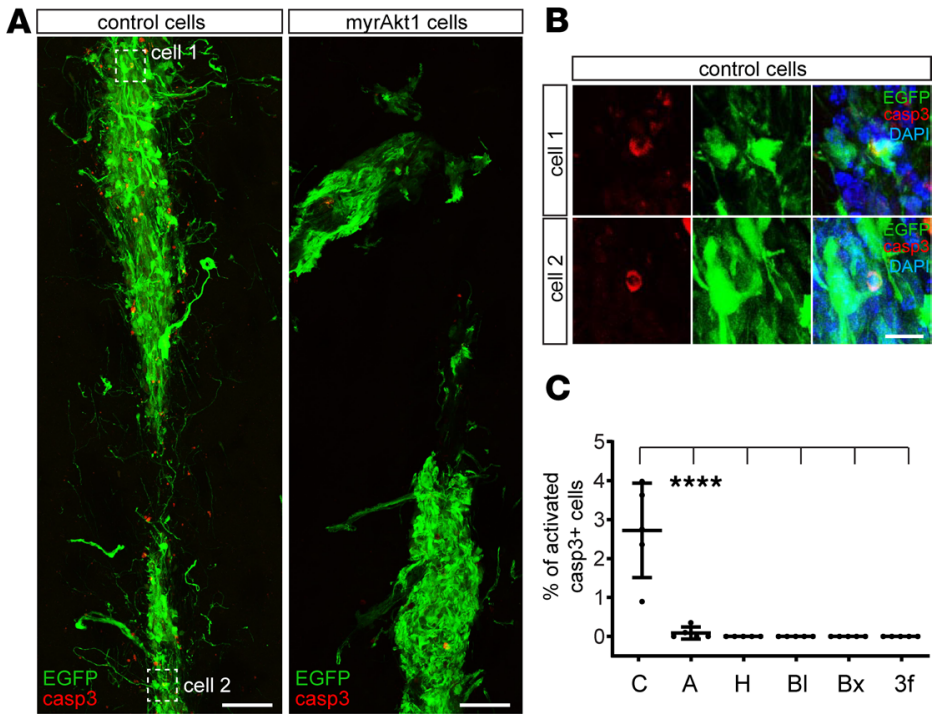

Figure 2. Genetic modification of $\mathbf{H 9}$ hNSCs abrogates their cell death in vivo. (A) Control and Akt1-expressing cells (5 days after transplantation). (B) Magnified images of control cells, coexpressing EGFP and activated caspase-3. (C) Percentage of transplanted cells expressing activated caspase-3 ( $n=5$ controls; $n=5$ genetically modified for each time point). Note that for $\mathrm{H}, \mathrm{BI}, \mathrm{Bx}$ and $\mathrm{3f}$ conditions we did not find any cells expressing activated caspase-3. Mean $\pm \mathrm{SD}$, Kruskal-Wallis with Dunn's post hoc tests, ${ }^{* * *} P<0.0001$. Scale bars: $100 \mu \mathrm{m}(\mathbf{A}), 20 \mu \mathrm{m}$ (B).

modification could also be reproduced in immunosuppressed wild-type mice that received either enhanced GFP-only (EGFP-only) or Akt1-expressing transplants of H9 hNSCs (Supplemental Figure 2).

To analyze whether a combination of prosurvival genes could enhance survival of transplanted cells better than a single prosurvival gene, we induced expression of the 3 most efficient prosurvival factors, namely, $A k t 1, B c l 2$, and Bcl2l1, in H9 hNSCs and transplanted genetically modified cells in the NSG mice. Strikingly, 3-factor (indicated as " $3 \mathrm{f}$ " in Figure 1E and Supplemental Figure 1) stimulation had effects on cell survival similar to those of myrAkt1 alone, indicating that all 3 factors most likely act via the same signaling pathway.

To confirm that expression of prosurvival genes decreases cell death, we analyzed the apoptotic marker activated caspase- 3 in transplanted cells. At 5 days posttransplantation, control transplants contained many activated caspase-3-positive cells, and some of them still expressed EGFP (Figure 2, A-C). Notably, expression of the apoptotic marker in genetically modified cells was hardly detectable (Figure 2, A-C).

Genetically modified transplanted H9 hNSCs exit the cell cycle and differentiate. Uncontrolled proliferation of transplanted cells can lead to tumor formation; therefore, we investigated how soon transplanted cells exit the cell cycle. To this end, we colabeled transplanted H9 hNSCs with Ki-67, a marker that was expressed only in cells that reentered the cell cycle. Specificity of Ki-67 labeling was confirmed by strong labeling of cycling neural precursor cells in the subventricular zone (SVZ) (Figure 3, A and B), a site of postnatal neurogenesis in mice $(20,21)$. By 1 month posttransplantation, only very few Ki- $67^{+}$cells were found in the transplants (Figure 3A). Furthermore, these cells often did not coexpress EGFP or human nucleus antigen (Figure $3 \mathrm{C}$ ), suggesting that a portion of $\mathrm{Ki}-67^{+}$cells represent endogenous glial cells that were activated upon transplantation. Indeed, among the genetically modified cells that expressed Hifla, Bcl2, or Bcl-xl, Ki-67 was expressed in only 1 out of 1000 cells by 3 months after transplantation (Figure 3, D and E). Importantly, those cells that expressed $A k t 1$ included a significantly higher number of Ki-67+ cells (Figure $3 \mathrm{E}$ ), and 3-factor-expressing cells showed a similar number of $\mathrm{Ki}-67^{+}$cells, indicating that these cells resemble cells that express $A k t 1$ only (Figure 3E). Thus, it is possible that the number of surviving myrAkt $1^{+}$cells is somewhat overestimated because some transplanted cells might continue to proliferate. Additionally, newly generated Akt1-expressing cells might replace some dying cells in the transplant, since in spite of a relatively high number of $\mathrm{Ki}-67^{+}$cells, Akt1-expressing grafts did not grow with time. Moreover, we never observed teratomas arising from Akt1-expressing transplants within the time frames of our analysis.

To analyze whether expression of prosurvival genes changes the differentiation profile of transplanted cells, we colabeled transplants with markers for immature and mature neural cells. All genetically modified 

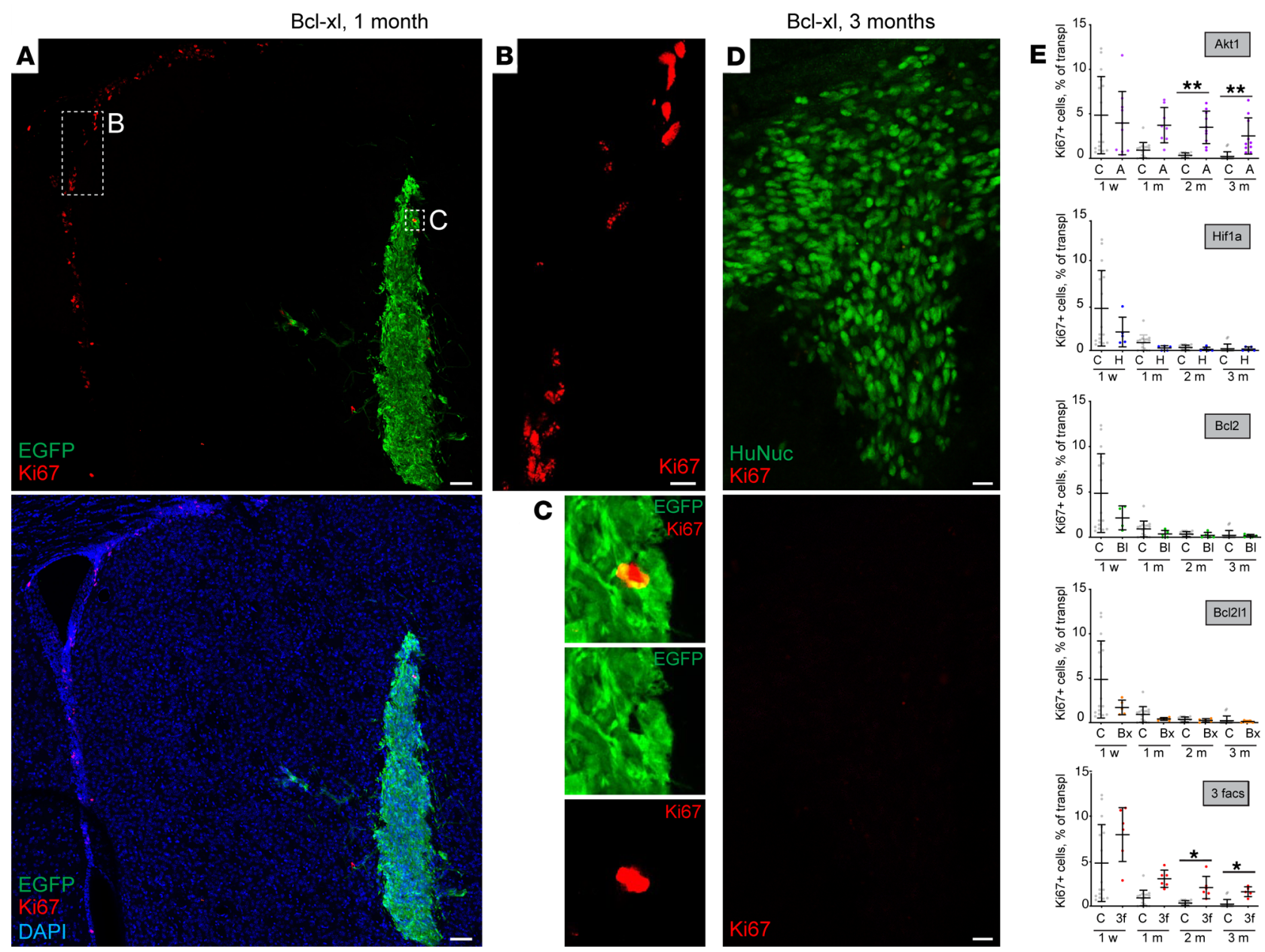

Figure 3. Genetically modified H9 hNSCs exit the cell cycle soon after transplantation. (A-C) Transplanted Bc/2/1-expressing H9 hNSCs 1 month posttransplantation stained with cell cycle marker Ki-67. Note that while Ki-67 robustly labels postnatal neurogenesis in the SVZ (B), very few cells in the transplant are labeled by Ki-67, and those are often not EGFP+, thus representing endogenous glial cells rather than transplanted cells. (D) Transplanted Bc/2/1-expressing cells 3 months posttransplantation. (E) Percentage of Ki-67+ cells in transplanted cells across different conditions 1 week and 1, 2, and 3 months after transplantation ( $n=14-16$ controls; $n=5-10$ genetically modified for each time point). Mean \pm SD, Kruskal-Wallis with Dunn's post hoc tests, only significant values are shown: ${ }^{*} P<0.05 ;{ }^{*} P<0.01$. Scale bars: $100 \mu \mathrm{m}(\mathbf{A}), 20 \mu \mathrm{m}$ (B and D).

transplants except those expressing Hifla contained higher numbers of nestin ${ }^{+}$cells, a marker of neural precursor cells, than controls at 3 months posttransplantation (Figure 4A). However, as shown above, in the case of Bcl2 and Bcl2l1 expression, it did not affect the number of $\mathrm{Ki}-67^{+}$cells (see Figure $3 \mathrm{E}$ ). Thus, although approximately $10 \%$ of $B c l 2$ - and $B c l 2 l 1$-expressing cells still continued to express nestin at 3 months posttransplantation, these cells did not express Ki-67 and had exited the cell cycle. Strikingly, only Bcl-xl enhanced neuronal differentiation as indicated by colabeling with NeuN, a neuronal differentiation marker (Figure 4B). Interestingly, in neuronal differentiation, 3-factor-expressing transplants resembled Bcl2l1expressing cells rather than Akt1 cells (Figure 4B). Both Bcl2l1 and Akt1 expression also enhanced oligodendrocyte differentiation (Figure $4 \mathrm{C}$ ), and none of the transplanted cells differentiated into glial fibrillary acidic protein-positive $\left(\mathrm{GFAP}^{+}\right)$astrocytes, at least by 3 months after transplantation (Figure 4D).

Short-term induction of expression of a prosurvival factor enhances long-term survival of transplanted H9 hNSCs. In some instances, it is not desirable in the long term to stimulate expression of a certain factor when sustained signaling by the factor triggers unwanted effects, e.g., enhanced proliferation or retarded differentiation. To control expression of transgenes in genetically modified H9 hNSCs, we designed an inducible lentiviral vector (Figure 5A). It had 2 parts, 1 expressing rtTA3 under the constitutively active promoter EF1 and the other coding for a gene of interest linked to EGFP via a T2A sequence expressed under the tetracycline response element-responsive, or "Tet-On," promoter. Thus, upon addition of Dox, rtTA3 activates the Tet- 
A

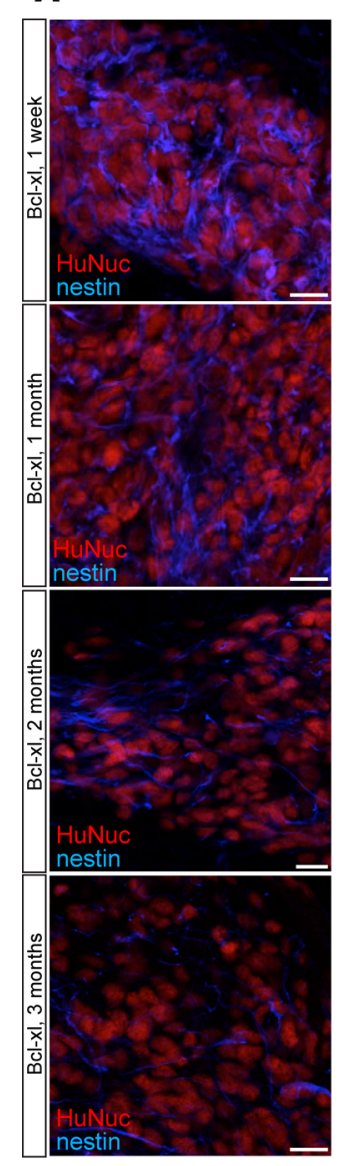

B
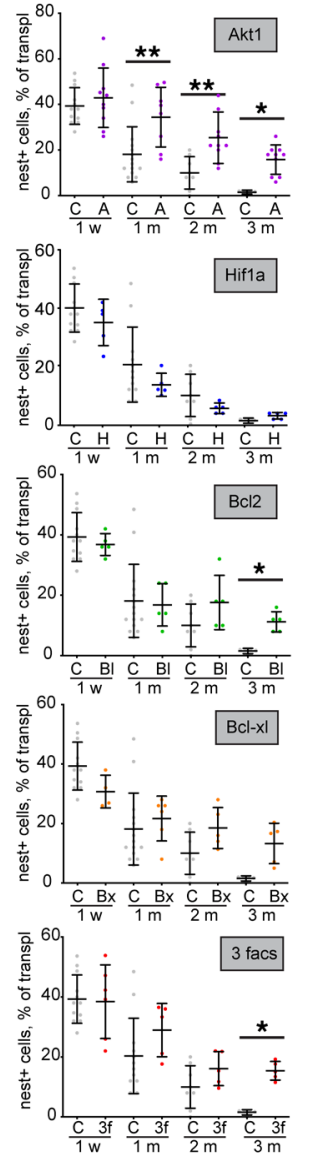

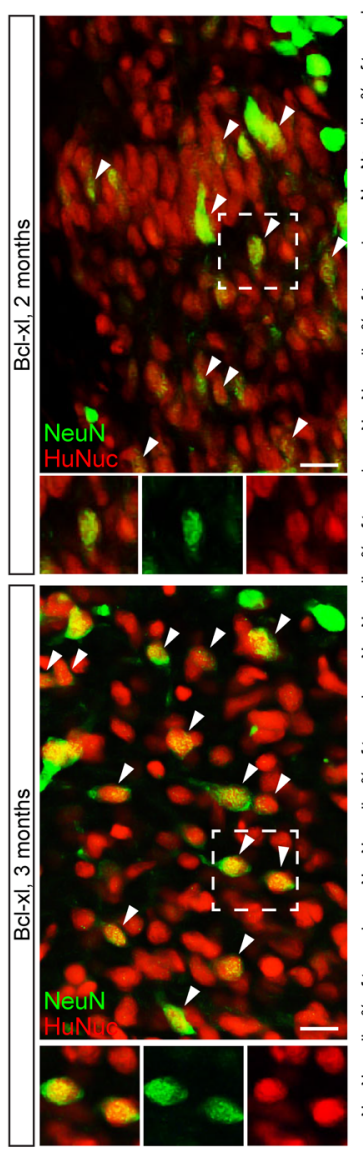

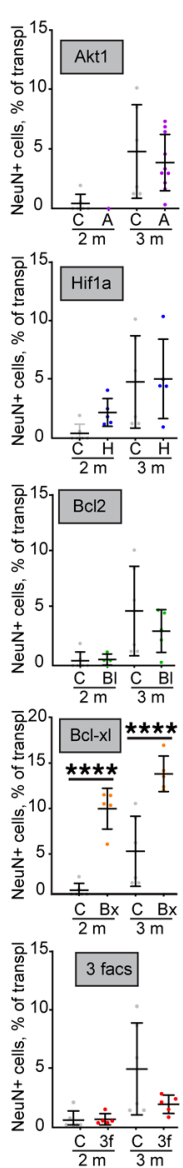

\section{C}
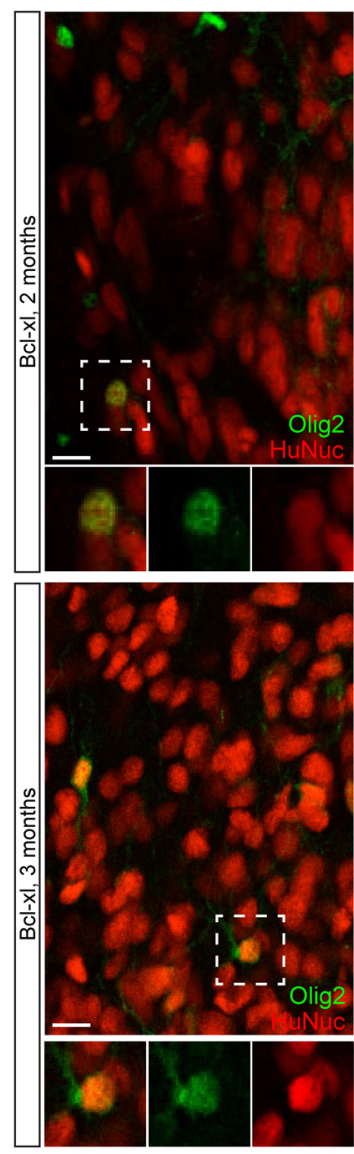

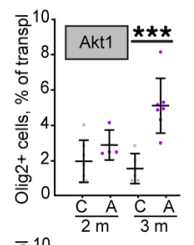

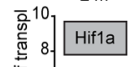

:

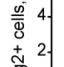

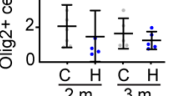

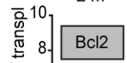

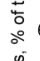

糖 4.

常到主主

言0 $\frac{C \text { Bl }}{\frac{C}{2 m} \frac{C B}{3 m}}$

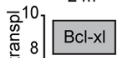

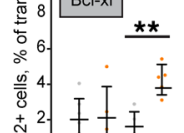
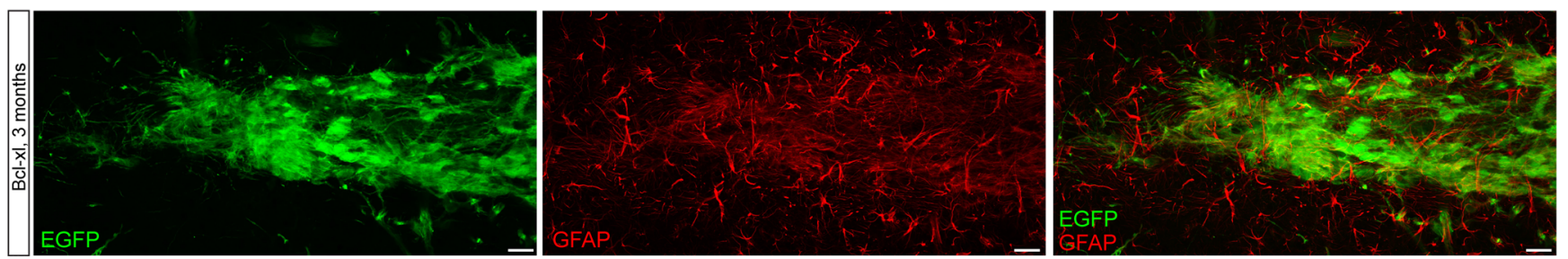

Figure 4. Transplanted genetically modified H9 hNSCs progressively differentiate into neural cells of neuronal and oligodendroglial fate. (A) Representative images of transplanted cells expressing Bc/2/1 and stained with nestin, a marker of neural precursor cells. Percentage of nestin ${ }^{+}$cells in transplanted H9 hNSCs, 1 week and 1, 2, and 3 months after transplantation ( $n=5-12$ controls; $n=5-10$ genetically modified). (B) Representative images of transplanted cells expressing Bcl2/1 and stained with NeuN, a marker of mature neurons. Percentage of NeuN ${ }^{+}$cells in transplanted H9 hNSCs 2 and 3 months after transplantation ( $n=5-6$ controls; $n=5-7$ genetically modified). Arrowheads indicate NeuN ${ }^{+} / \mathrm{HuNuc}^{+}$cells. (C) Representative images of transplanted cells expressing Bcl2/1 and stained with Olig2, a marker of oligodendrocytes. Percentage of Olig2 ${ }^{+}$cells in transplanted cells 2 and 3 months after transplantation ( $n=5-7$ controls; $n=5-7$ genetically modified for each time point). (D) Transplanted genetically modified H9 hNSCs do not differentiate into astrocytic lineage in 3 months as evidenced by lack of coexpression of astrocytic marker GFAP. Mean \pm SD, 1-way ANOVA with Holm-Šídák post hoc tests, only significant values are shown: ${ }^{*} P<0.05 ;{ }^{* *} P<0.01 ;{ }^{* *} P<0.001 ;{ }^{* * *} P<0.0001$. Scale bars: $20 \mu \mathrm{m}$ (A-D).

On promoter and drives the expression of the gene of interest together with EGFP. We tested this lentivirus in vitro by transducing H9 hNSCs and activating EGFP expression with Dox (Figure 5B). Without Dox, EGFP was not expressed in the transduced cells. However, 1 day after adding Dox to the cell medium, strong EGFP expression was observed in every cultured cell. Two days after withdrawing Dox, EGFP expression was weak and disappeared by 8 days after withdrawal (Figure 5B).

We further confirmed applicability of the lentivirus in vivo by directly injecting it into the SVZ of adult mouse brains. It is known that neuroblasts produced in the SVZ migrate toward the olfactory bulb and mature there into interneurons $(13,22)$. To label these cells, we put Dox in the drinking water of the mice 20 days after injection and analyzed EGFP expression (Figure 5C). The olfactory bulb did not contain any 
A
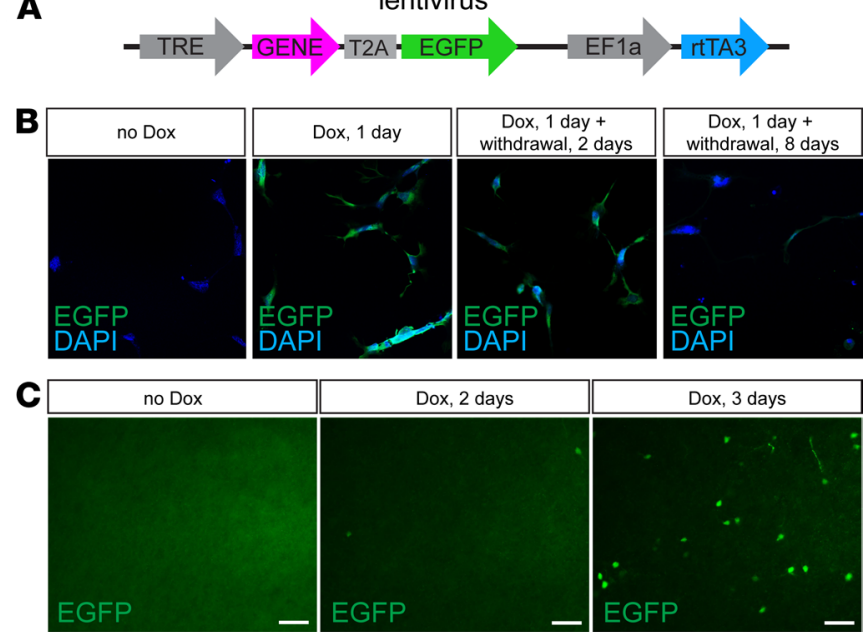

D
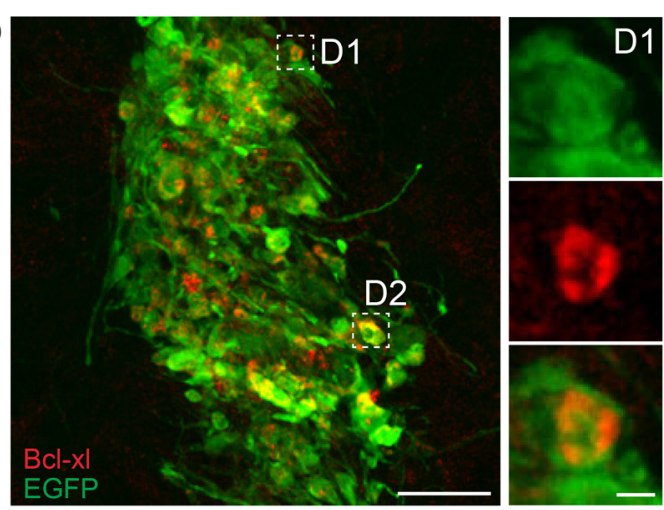

E

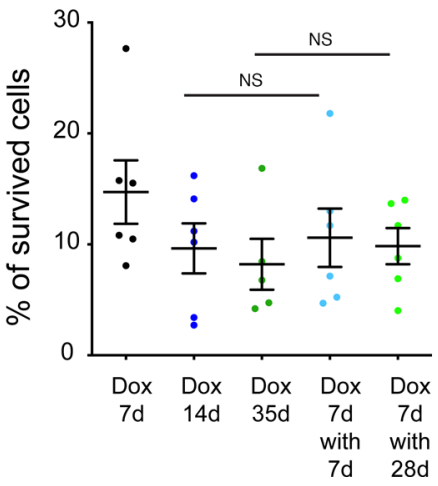

Figure 5. Temporal activation of a prosurvival gene leads to long-term enhancement of survival of transplanted H9 hNSCs. (A) Lentiviral vector construct with Dox-inducible transgene expression. (B) H9 hNSCs infected with EGFP-coding lentiviral vector without Dox, 1 day of Dox activation, 1 day of Dox activation followed by 2 days of Dox withdrawal, and 1 day of Dox activation followed by 8 days of Dox withdrawal. (C) Dox-regulated EGFP-coding lentivirus was injected into the SBZ. Representative images of the olfactory bulb 20 days posttransplantation, when lentivirus-infected neuroblasts had already migrated to the olfactory bulb. Mice were given Dox and EGFP+ neurons in the olfactory bulb were labeled,

before Dox was given to mice and after 2 days and 3 days of Dox activation. (D) H hNSCs were transduced by the lentivirus with Dox-activated expression of $B c / 2 / 1$ and EGFP and coexpressed $B c / 2 / 1$ and EGFP 7 days posttransplantation. (E) Percentage of cell survival for H9 hNSCs that were transduced by the lentivirus with Dox-activated expression of $B c / 2 / 1$ and EGFP and transplanted in the striatum $(n=5-6)$; mice were treated with Dox for 7 days posttransplantation (Dox 7d), Dox for 14 days (Dox 14d), Dox for 35 days (Dox 35d), Dox for 7 days followed by Dox withdrawal and 7 days after withdrawal (Dox 7d with 7d), or Dox for 7 days followed by Dox withdrawal and 28 days after withdrawal (Dox 7d with 28d). Mean \pm SD, 1-way ANOVA with HolmŠídák post hoc tests. Scale bars: $50 \mu \mathrm{m}$ (C and D), $5 \mu \mathrm{m}$ (insets).

$\mathrm{EGFP}^{+}$cells before Dox treatment, whereas by 3 days on Dox, numerous EGFP ${ }^{+}$cells could be detected (Figure 5C). Thus, both in vitro and in vivo experiments provided evidence that, in the infected cells, expression of the gene of interest could be regulated using our inducible vector system.

To analyze whether transient expression of a prosurvival gene enhances transplanted cell survival similarly to constitutive expression, we transduced H9 hNSCs with a lentivirus expressing rtTA3 and Bcl2l1 together with EGFP. We transplanted genetically modified H9 hNSCs into the striatum of NSG mice that were pretreated for 3 days with Dox. At 7 days posttransplantation, all H9 hNSCs expressed EGFP and Bcl-xl (Figure 5D). Importantly, survival of transplanted H9 hNSCs that exhibited Dox-regulated Bcl2l1 expression (Figure 5E) was comparable to those H9 hNSCs with permanent Bcl2ll expression (Figure 1E). There was a slight decrease in survival of Bcl2l1-expressing H9 hNSCs at 1 month posttransplantation in mice that continuously received Dox (Figure 5E), similar to permanent Bcl-xl overexpression. However, and most importantly, Dox withdrawal at 7 days posttransplantation did not affect survival of transplanted cells analyzed either 7 days or 28 days after withdrawal (Figure 5E). Thus, upregulation of the prosurvival pathway for just 7 days after transplantation is enough to trigger long-term survival of transplanted cells. 
Genetic modulation of survival can be applied to hNSCs from different sources. To investigate whether modulation of prosurvival factors is applicable to hNSCs in general, we differentiated hNSCs from a clinically relevant human embryonic stem cell (hESC) line (RC17), which has been derived under good manufacturing practice. The RC17-derived hNSCs were maintained after differentiation under the same culturing conditions as had been used for the H9 hNSCs, and we confirmed that the RC17 hNSCs expressed markers of forebrain NSCs: orthodenticle homeobox 2 (OTX2), paired box 6 (PAX6), Sry-related box 1 (SOX1), and N-cadherin (Figure 6, A and B). From the study with H9 NSCs, we found Bcl-xl and Bcl-2 to be the most promising factors for hNSC survival modulation in terms of translational potential, because they provided a strong and persistent prosurvival effect accompanied by fast cell cycle exit. Therefore, we genetically modified RC17 hNSCs with lentiviruses to overexpress either Bcl2l1 or Bcl2 and compared them with RC17 hNSCs expressing EGFP only. Similar to H9 hNSCs, all infected RC17 hNSCs expressed EGFP, indicating a complete transduction (Figure 6C). Transduced RC17 hNSCs were cultured for 4 days without growth factors followed by transplantation into the striatum of immunodeficient NSG mice. Control cells were transplanted into the left and genetically modified cells into the right striatum (Figure 6A). Strikingly, the prosurvival effect of both $B c l 2 l 1$ and $B c l 2$ was even stronger for RC17 hNSCs than for H9 hNSCs. Thus, intrinsic survival potential of RC17 hNSCs was much lower than of their H9 hNSC counterparts - only $0.62 \%$ and $0.14 \%$ of control RC17 hNSCs survived 1 week and 1 month posttransplantation, respectively (Figure 6, D and E), compared with approximately 7\% and approximately 5\% for $\mathrm{H} 9 \mathrm{hNSCs}$ (Figure 1, D and E). Furthermore, already at 1 month posttransplantation, we noticed complete cell death of 2 control RC17 hNSC transplants, which was not observed for $\mathrm{H} 9$ hNSCs until 3 months posttransplantation. Nevertheless, modulation of Bcl2l1 or Bcl2 expression increased survival of RC17 hNSCs to levels that were similar to H9 hNSCs (Figure 6, D and E). Thus, by calculating fold increase in survival, we show a 147- and 131-fold enhancement in 1-month survival of transplanted RC17 hNSCs by modulating $B c l 2$ and Bcl2l1 expression, respectively.

To analyze whether an active cell cycle could contribute to such a dramatic prosurvival effect, we labeled transplanted RC17 hNSCs with anti-Ki-67 antibodies. As before, the specificity of Ki-67 labeling of actively cycling cells was confirmed by detection of cycling neural precursor cells in the SVZ (Figure 6F). By 1 month posttransplantation, very few Ki- $67^{+}$cells were found in the RC17 transplants (Figure 6, F and $\mathrm{G})$, indicating that modulation of $B c l 2$ or $B c l 2 l 1$ expression has a truly prosurvival effect, with little impact on additional proliferation.

In summary, all major effects of prosurvival modulation have been reproduced using RC17-derived hNSCs, including a dramatic increase in short- and long-term survival of genetically modified transplanted cells and exit from the active cell cycle.

Transplanted hNSCs with genetically enhanced survival have improved behavioral abnormalities in a mouse model of stroke. In order to investigate whether genetically enhanced survival of transplanted cells increases the impact of cell therapy in neurological disorders, we used a mouse model of mild stroke where the middle cerebral artery is permanently occluded (middle cerebral artery occlusion, MCAO) to induce ischemic brain damage. Such a distal MCAO model affects the cortex focally, sparing subcortical regions, and mimics the majority of human strokes that occur in the territory of the middle cerebral artery. In this model, genetically modified hNSCs that are transplanted into the infarct zone should not only positively influence the surrounding brain region but also resist the hostile necrotic environment of the infarct zone. Two days after MCAO, H9 or RC17 hNSCs transduced with the control lentivirus (EGFP-only) or lentivirus-expressing Bcl2l1 together with EGFP were transplanted into the peri-infarct zone in the brains (Figure 7A). A sham group received a sham injection after MCAO. In order to assess the effect of genetically modified cell transplants, the stroke-induced neurological deficit was evaluated using 3 sensorimotor function tests 1 day before MCAO and 7 days after cell transplantation. Importantly, both $\mathrm{H} 9$ and $\mathrm{RC} 17 \mathrm{hNSCs}$ with enhanced survival led to significant functional improvements in MCAO-affected mice relative to sham and unmodified H9 and RC17 hNSCs (Figure 7, B-Q). Furthermore, although there were some specific differences between effects of $\mathrm{H} 9$ and RC17 hNSCs on enhanced survival, the overall effect was similar for both types of hNSCs. Thus, both H9 and RC17 Bcl2l1-expressing cells provided a rather minor effect on functional improvement in the latency-to-move (Figure 7, B-E) and sticky-tape tests (Figure 7, F-I), and the effect was larger for RC17 Bcl2l1-expressing cells.

In contrast to previous reports (23), distal MCAO had no effect on sham-injected mice regarding the sticky-tape and latency-to-move test. Such a lack of effect might be due to depletion of the immune system in the NSG mice because the response of the immune system to an ischemic insult is crucial for development of the injury (24-26). However, a clear stroke-induced deficit in sensorimotor function was detected 
A

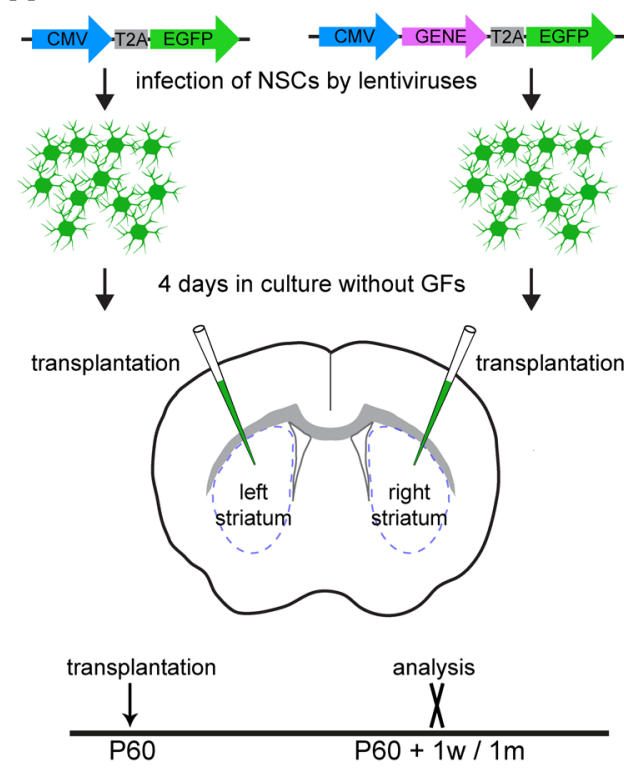

D

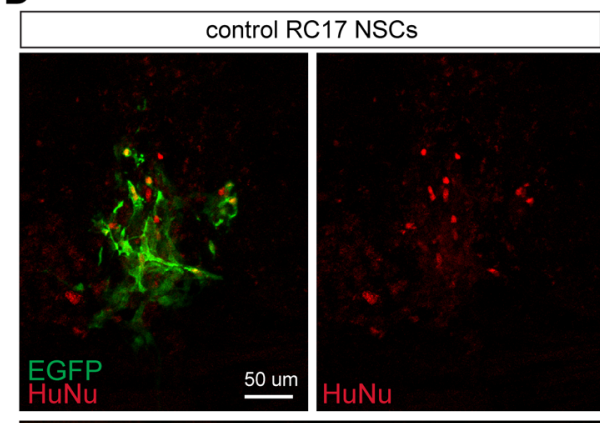

$\mathbf{F}$

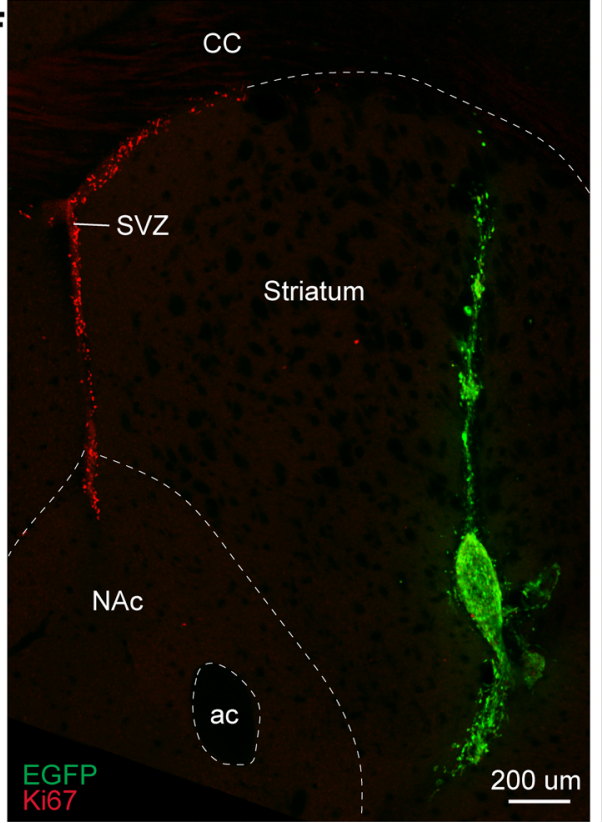

B
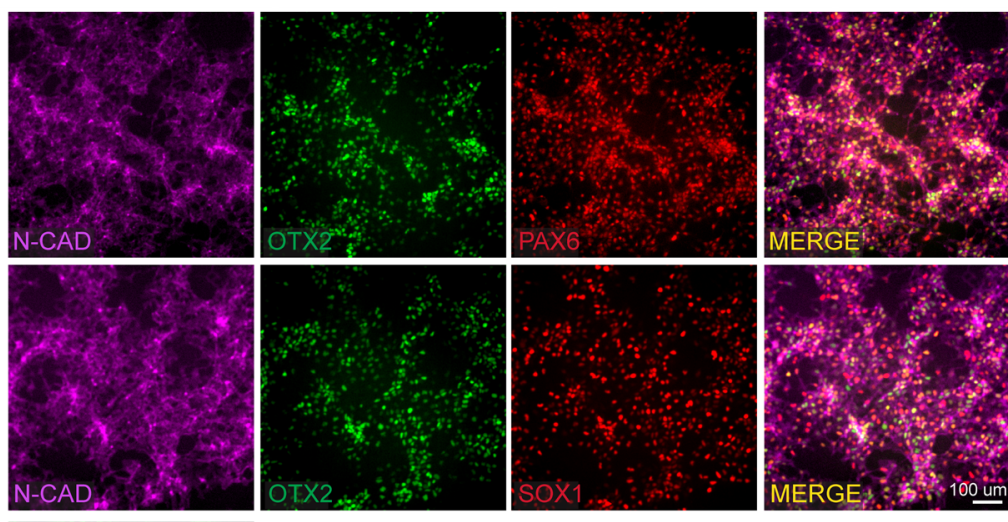

MERGE 2 os: 100 im

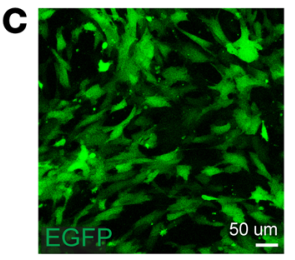

E

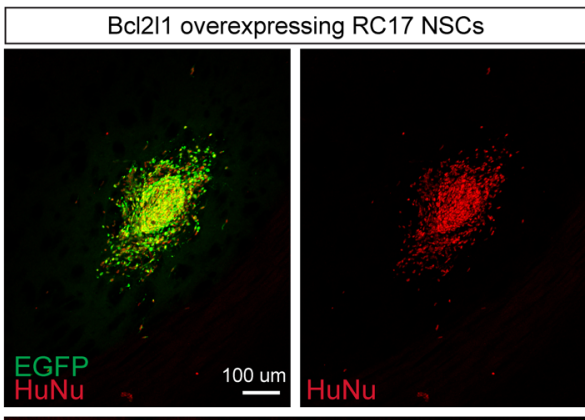

CC

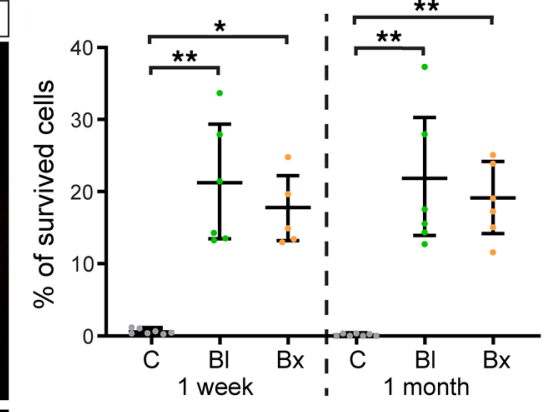

G

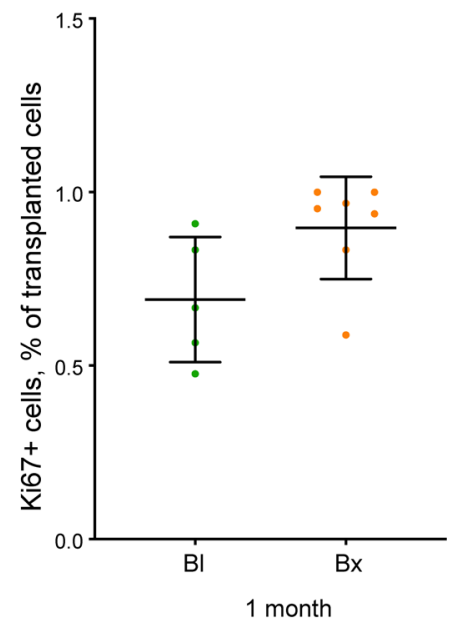

Figure 6. Genetic modification of another hNSC line that is derived from RC17 ESCs reproduces a prosurvival effect upon transplantation. (A) Cultured RC17 NSCs were infected with pCDH-CMV-MCS-T2A-EGFP lentivirus, empty or expressing Bc/2 or Bc/2/1 genes. Cells were incubated 4 days in the medium without growth factors and then transplanted into the striatum of 60-day-old NSC mice: control cells into the left and genetically modified cells into the right striatum, respectively. Transplants were analyzed 1 week and 1 month posttransplantation. (B) Differentiation of RC17 hESCs into NSCs was assessed by labeling of N-cadherin (neural progenitor marker), OTX2 (forebrain and midbrain marker), PAX6 (dorsal neural progenitor marker), and SOX1 (neural progenitor marker) staining of RC17 NSCs on day 15 in cell culture. (C) RC17 hNSCs infected by pCDH-CMV-MCS-T2A-EGFP lentivirus 4 days after infection. (D) Control and BcI2/1-overexpressing RC17 hNSCs 1 month after transplantation. Note different scale bars (larger scale bar for control RC17 hNSCs to show few surviving cells). (E) Estimation of 
RC17 hNSC survival (percentage of total transplanted cells), 1 week and 1 month after transplantation ( $n=7$ controls; $n=5-6$ genetically modified for each time point). (F) Transplanted Bc/2/1-expressing RC17 NSCs 1 month posttransplantation stained with cell cycle marker Ki-67. Although Ki-67 robustly labels postnatal neurogenesis in the SVZ, very few cells in the transplant are labeled by Ki-67. (G) Percentage of Ki-67+ cells in transplanted cells 1 month after transplantation (n $=2$ controls; $n=7$ genetically modified). Note that naive RC17 hNSCs have very low survival that often precludes reliable quantification because of too few (or lack of) surviving cells. Mean \pm SD, 1-way ANOVA with Dunn's post hoc tests. ${ }^{*} P<0.05 ;{ }^{* *} P<0.01$. Scale bars: $100 \mu \mathrm{m}$ (B and D), $50 \mu \mathrm{m}$ (C), and $200 \mu \mathrm{m}$ (F).

using a handedness test. Although the number of grabbing attempts with the contralateral paw and the Collins score were clearly reduced in sham and EGFP-only groups, mice with Bcl2l1-expressing H9 or RC17 cell transplants completely recovered to baseline level in a week after transplantation (Figure 7, J-Q). Importantly, the transplantation of control cells had no or a very minor effect on recovery in the handedness test (Figure 7, J-Q). Thus, Bcl2l1-expressing hNSCs of both types successfully promoted the recovery of damaged brain areas responsible for coordinating the forelimbs.

To validate that expression of a prosurvival gene increases survival of transplants in a hostile environment of brain tissue affected by stroke, we analyzed the transplantation area immediately after the behavioral tests. Because all brains in MCAO models were missing a large part of the cortex in the area of ischemia because of massive cell death (Figure 8A, damaged area), we quantified the number of transplanted cells surrounding the damaged area. Importantly, while only a few control hNSCs were found, genetic modulation of hNSCs dramatically increased the number of hNSCs that survived around the damaged area (Figure 8, A-D; only RC17 hNSCs are shown because quantifications could not be done for $\mathrm{H} 9$ hNSCs; see Methods). Because we transplanted cells in or near the infarct zone, we expect a large part of hNSCs, being settled in fragile tissue in the epicenter of damage, could not be recovered during histology. Thus, the actual number of survived hNSCs might be much larger than we observed. Overall, we demonstrated that genetically modified hNSCs were better protected from the hostile environment of the infarct zone than naive cells, allowing genetically modified cells to have a stronger effect on recovery from injury in neurological disorders.

\section{Discussion}

Poor survival of transplanted cells in brain transplantations decreases the efficacy of cell therapy and requires large numbers of transplanted cells to be generated. Here, we demonstrate that survival of transplanted hNSCs can be dramatically improved by stimulating prosurvival pathways, leading to better functional recovery than that of naive hNSCs in a brain disease model.

Numerous studies show poor survival of transplanted hNSCs in the brain, and often the numbers of cells derived from the transplant do not reflect the pure survival potential of hNSCs, but rather the replenishment and expansion of the cell population due to extensive proliferation of hNSCs, which may result in tumor formation $(3-8,27)$. In the survival of transplanted cells, 2 phases can be distinguished: so-called short-term survival (or immediate death), which ends by 1 week posttransplantation, and a long-term period (continuous cell death) that persists for months. In our experiments, all 4 prosurvival factors profoundly inhibited the caspase-3-dependent apoptotic pathway. Expression of Akt1, Bcl2, and Bcl2l1 not only significantly protected transplanted cells from immediate death but also abrogated continuous cell death, while Hifla expression delayed immediate cell death only. Interestingly, the number of Hifla-expressing transplanted cells did not drop between 1 and 3 months after transplantation, indicating that Hifla expression protected against continuous cell death. Expression of Hifla was also reported to improve survival of mouse NSCs that were transplanted in the brain ventricle (28).

Because nondifferentiated NSCs can become tumorigenic after transplantation $(7,8)$, we were concerned about possible tumorigenesis in the animals transplanted with hNSCs expressing prosurvival factors. In order to decrease the proliferating potential of hNSCs, we initiated cell differentiation directly in the culture by withdrawing growth factors from the medium. Importantly, for 3 out of 4 prosurvival factors, fewer than $0.5 \%$ of $\mathrm{Ki}-67^{+}$ cells were observed at 1 month after transplantation and approximately $0.1 \%$ at 3 months after transplantation.

Availability of prosurvival factors varies within the brain, and the response to different prosurvival factors depends on the stages of neuronal maturation (for a review, see ref. 12). Thus, we chose 4 prosurvival factors, Hifla, Akt1, Bcl2, and Bcl-xl, which have the potential to stimulate survival of neural cells very early during cell differentiation. Akt1 signaling regulates many cellular processes, including prosurvival mechanisms, via regulation of a large number of substrates, such as p53, mammalian target of rapamycin (mTOR), and caspase-3/caspase-9, thus contributing to antiapoptotic signaling in various cell types $(29,30)$. 


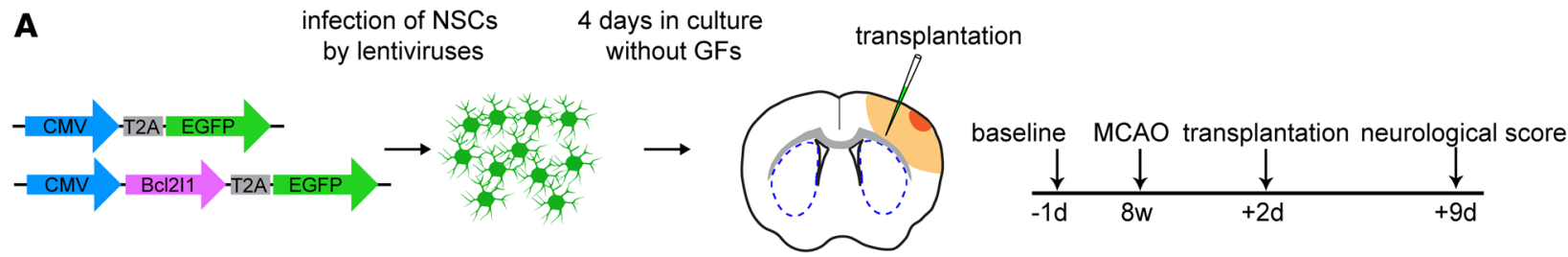

H9 NSCs

RC17 NSCs

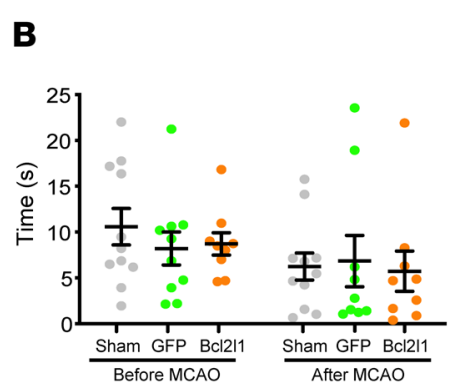

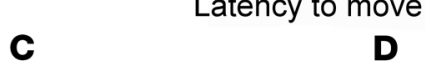
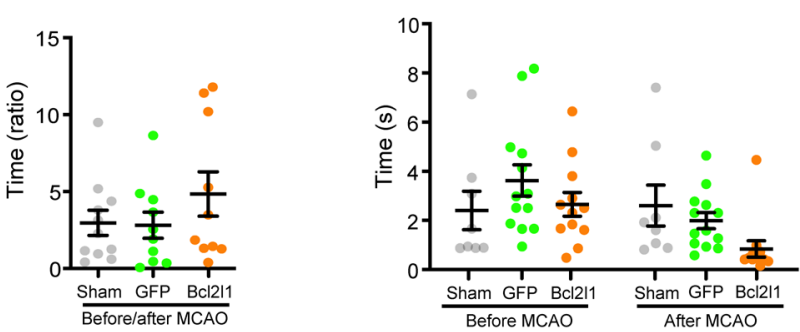

E

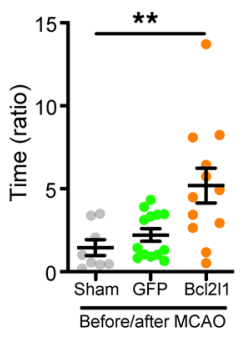

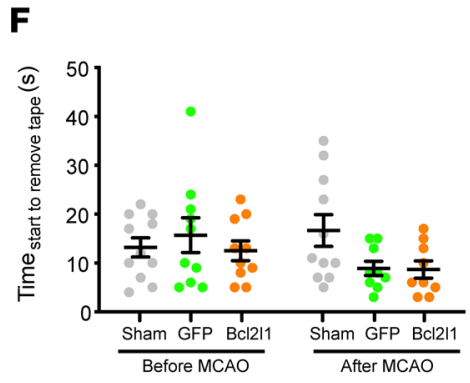
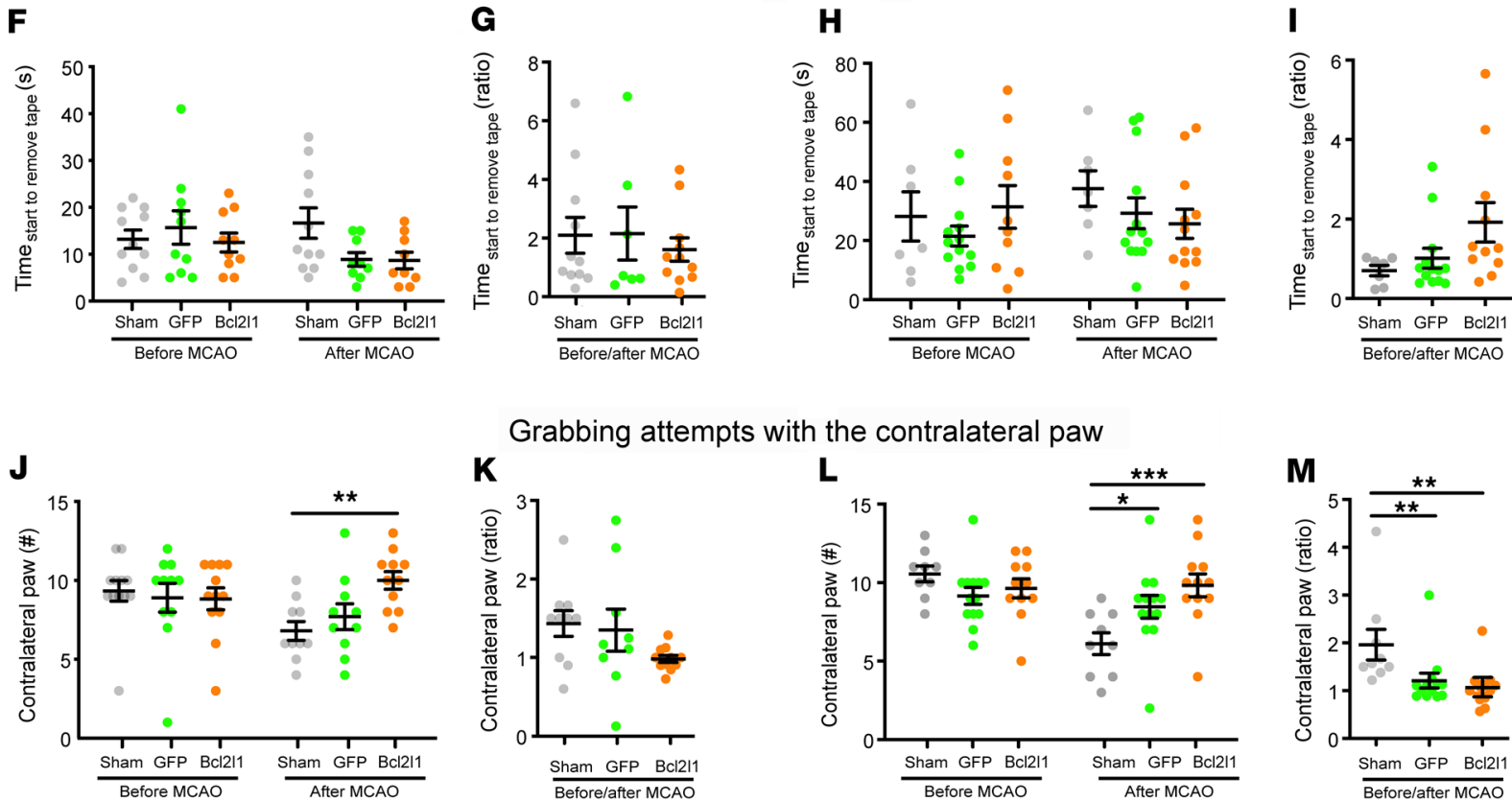

Grabbing attempts with the contralateral paw
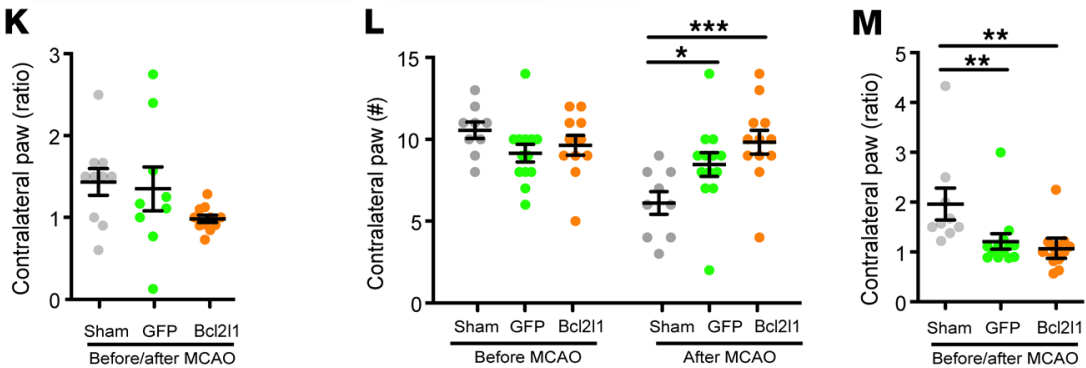

\section{Collins score}
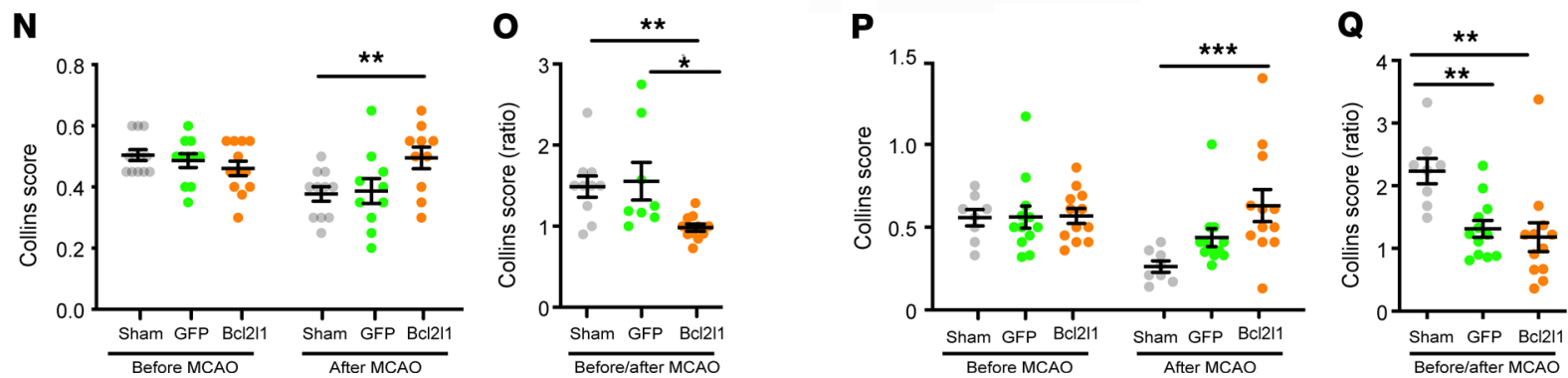

Figure 7. Genetically modified hNSCs improve ischemia-induced neurological deficits. (A) Two days after MCAO, EGFP-only or Bc/2/1-expressing H9 or RC17 NSCs were injected into the peri-infarct area, and neurological score was estimated 7 days later. (B-E) Latency-to-move test. Bc/2II-expressing RC17 NSCs significantly improved mobility in latency-to-move test compared with sham, indicated by the ratio before and after MCAO. (F-I) Sticky-tape test. No significant differences before and after MCAO as well as between sham, EGFP, or Bc/2/1 could be detected using sticky tape. (J-Q) The handedness of mice using Collins test. Transplantation of $\mathrm{H} 9$ and RC17 hNSCs with enhanced Bc/2/1-mediated survival increased the number of grabbing attempts with 
the contralateral paw compared with sham, thus improving behavior outcome to the level before MCAO (H9, J, 2-way ANOVA for interaction, $F_{(2 / 60)}=3.51$; RC17, L, 2-way ANOVA for interaction, $\left.F_{(2 / 61)}=6.22\right)$. RC17 hNSCs also significantly affected the ratio of grabbing attempts before and after MCAO (M). Both Bc/2/1-expressing $\mathrm{H} 9$ and RC17 hNSCs improved Collins score in comparison to sham-expressing hNSCs and H9 also in comparison to EGFP-expressing hNSCs, reaching a pre-MCAO level (H9, N, 2-way ANOVA for interaction: $F_{(2 / 60)}=4.96 ;$ RC17, $\mathbf{P}, 2$-way ANOVA for interaction, $\left.F_{(2 / 58)}=3.41\right)$. The ratio of the Collins scores before and after MCAO was improved by transplantation of Bcl2/1-expressing hNSCs compared with sham-expressing and, in the case of H9, with EGFP-expressing hNSCs. For B, D, F, H, J, L, N, and P, mean \pm SEM, 2-way ANOVA, Bonferroni's posttest. For C, E, G, I, K, M, O, and Q, mean \pm SEM, 1-way ANOVA, Tukey's post hoc test. ${ }^{*} P<0.05$; ${ }^{*} P<0.01 ;{ }^{*}{ }^{*} P<0.001 . n=9-12$.

Extensive data show that Akt1 regulates prosurvival signaling even in precursors, not only in immature neurons (31-33). For instance, mTOR, which is regulated by Akt1 (30), controls cell cycle length and exit in several types of cells, including neuronal progenitor cells, and knockout of Mtor results in a dramatic decrease in numbers of precursor cells (34). However, it may be difficult to distinguish between the regulation of cell death and other intracellular pathways, and increased expression of Akt1 can result in too broad of a spectrum of effects, depending on current cell fate and the environment surrounding cells. Furthermore, although in our study Akt1 overexpression resulted in a dramatic increase in transplanted cell number, Akt1-expressing cells stayed in active cell cycle for much longer than other genetically modified cells, thereby diminishing the translational potential of Akt1-expressing cells because of potential teratogenic effects.

The Bcl-2 protein family acts as key regulators in the intrinsic or mitochondrial apoptosis pathway. The family comprises 2 types of members: those that prevent apoptosis (Bcl-2 and Bcl-xl) (35) and those that promote apoptosis (Bax, Bid, and Hrk) (35). Systemic delivery of Bcl-xl fusion protein inhibited caspase-3 and -9 activities and prevented translocation of apoptosis-inducing factor into the nucleus following ischemia/reperfusion brain injury (36). In our experiments, both Bcl2- and Bcl2l1-expressing transplanted cells exhibited a high survival rate, and the transplanted cells exited the cell cycle soon after the transplantation. $B c l 211$, but not $B c l 2$, expression also enhanced neuronal differentiation of the transplanted cells. Interestingly, a recent study showed that $B c l 2$ expression also protected cortical neuron progenitors from cell death in vitro (37), suggesting that Bcl-2 family proteins are good candidates for neuroprotection at various stages of neuronal differentiation. Importantly, we showed in the current study that Bcl2l1 expression for only 1 week after transplantation protected transplanted neuronal progenitors for more than 1 month in vivo. The ability to regulate gene expression in the transplanted cells is one of the most critical issues for successfully implementing gene therapies. Our results demonstrate that short-term expression of a transgene in the transplanted cells could have long-lasting effects. A similar system can be used to quickly turn transgene expression "off" and "on" in transplanted cells when necessary, thus enhancing the safety of the cell therapy.

Cerebral ischemia is characterized by oxidative stress, an inflammatory response, and apoptosis in the infarct core and penumbra zone (62). Thus, it is an excellent model to test the potential of genetically modified hNSCs that are transplanted in the necrotic environment and experience strong prodeath signaling from the environment. The immediate, positive effect of transplanted NSCs after stroke is mediated by the secretion of trophic prosurvival factors affecting surrounding cells (38). However, a severe limitation of treating ischemic brain injuries with exogenous hNSCs is that only a small number of cells could survive after transplantation in the damaged tissue. Here, we injected hNSCs at 48 hours after distal, permanent MCAO in or near the peri-infarct zone. Very few control cells survived in the hostile environment, whereas survival of Bcl2l1-expressing hNSCs was 22-fold higher. Moreover, Bcl2l1-expressing but not control hNSCs improved the deficit in sensorimotor function, reflecting an improved outcome in these mice. Thus, results suggest that to achieve functional benefits from transplantation in the stroke model, many fewer genetically modified hNSCs could be transplanted (50,000 genetically modified cells instead of 1,000,000 naive cells by approximation of approximately 20 -fold increase in survival of Bcl2l1-expressing cells). Importantly, all major effects of prosurvival modulation were reproduced in 2 stem cell lines, $\mathrm{H} 9$ and RC17 hNSCs, which increases the robustness of the results and therapeutic relevance of our approach.

Stem cell therapy offers the possibility of a renewable source for replacing cells and tissues to treat a long list of diseases, conditions, and disabilities, including Parkinson's and Alzheimer's diseases, spinal cord injury, stroke, cerebral palsy, amyotrophic lateral sclerosis, loss of vision, and other neurodegenerative diseases. However, up to $98 \%$ of transplanted hNSCs die within 1 to 2 months after transplantation $(27,39)$. Therefore, tens of millions of cells are currently required for human cell transplantation therapies because of the low survival, which reduces the feasibility of cultivating such enormous numbers 

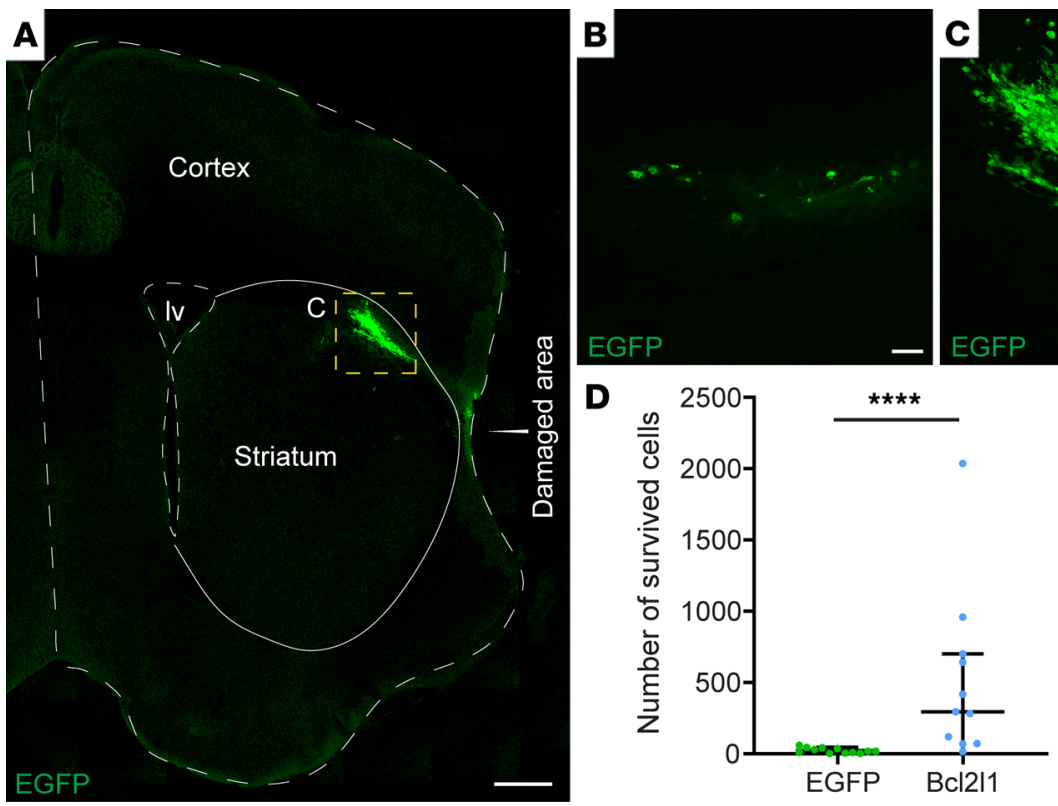

Figure 8. Bc/2/1 overexpression strongly enhances the survival of hNSCs after transplantation into the mouse brain with ischemic injury. (A) Grafted genetically modified RC17 hNSCs in the left hemisphere with ischemic injury (part of the brain is missing due to the massive cell death after MCAO, labeled by "Damaged area"). (B-C) Magnified images of control EGFP-expressing and Bc/2/1-expressing RC17 hNSCs, respectively. Scale bars: $500 \mu \mathrm{m}$ (B), 50 $\mu \mathrm{m}$ (C). (D) Number of transplanted RC17 hNSCs 9 days after MCAO, 7 days after transplantation. Median with IQR Mann-Whitney $U$ test. ${ }^{* * *} P<0.0001 . n=11$ controls; $n=12 B c / 2 / 1$ overexpressing.

of cells. Moreover, massive death of the transplanted cells will induce an additional inflammatory reaction in the injured brain areas, thus providing negative instead of positive effects for the diseased brain. Enhancing survival of transplanted cells in cell therapy for brain disorders would not only improve the outcome of cell therapy and avoid additional injury from the transplantations themselves but also dramatically decrease the number of cells needed to be generated for cell therapy.

\section{Methods}

Animals. For all the experiments, we used immunodeficient NSG mice (40) and wild-type C57BL/6 mice. All experimental procedures involving mice were performed according to the regulations of Copenhagen University and Lübeck University (Ministerium für Energiewende, Landwirtschaft, Umwelt, Natur und Digitalisierung, Kiel, Germany. Mice were housed under standard conditions (12-hour light/12-hour dark cycle, access to dry food and water ad libitum). Both males and females were used throughout the study.

Molecular subcloning. The $\mathrm{pCDH}$-CMV-MCS-T2A-EGFP vector was generated by modifying pCDH-EF1MCS-T2A-copGFP (System Bioscience), where the EGFP and CMV cassettes were subcloned from pEGFP-C1 (Clontech-Takara Bio) and the T2A sequence was subcloned from pCDH-CMV-T2A (41). The shuttle expression plasmids containing ORFs of mouse Akt1, Hifla, Bcl2, and Bcl2l1 (Bcl-xl protein) genes were ordered from Biocat and subcloned into the multiple cloning site of the pCDH-CMV-MCS-T2A-EGFP vector.

Recombinant lentivirus production and titration. Lentiviruses were produced as previously described (21). Human embryonic kidney 293 cells (HEK293 cells, Gibco, Thermo Fisher Scientific) were maintained in growth medium (DMEM, $2 \mathrm{mM}$ glutamine, 10\% FCS, 1\% penicillin/Streptomycin, all from Gibco, Thermo Fisher Scientific) and incubated at $37^{\circ} \mathrm{C}, 5 \% \mathrm{CO}_{2}$. The day before transfection, HEK293 cells were plated on $12 \mathrm{~cm} \times 10 \mathrm{~cm}$ plates $\left(4 \times 10^{6}\right.$ cells $/$ plate) so that the cells were approximately $80 \%$ confluent at the time of transfection. Growth medium was changed to transfection medium (DMEM, $2 \mathrm{mM}$ glutamine) 1 hour before transfection. The cells were transfected by using the calcium phosphate method with 4 plasmids: recombinant lentiviral expression vector $(10 \mu \mathrm{g} / \mathrm{plate})$ and 3 helper plasmids (pRev, pRRE, and MD2.G, $5 \mu \mathrm{g}$ of each helper per plate). Plasmid DNA was mixed with $\mathrm{CaCl}_{2}$ (final concentration $0.14 \mathrm{M}$ ) and $2 \times \mathrm{HBSS}$ (final: $280 \mathrm{mM} \mathrm{NaCl}, 1.5 \mathrm{mM} \mathrm{NaH}_{2} \mathrm{PO}_{4}\left[\times 2 \mathrm{H}_{2} \mathrm{O}\right], \mathrm{pH} 6.96$ ), with a final volume of $12 \mathrm{~mL}$ of the mixture. The mixture was added directly to transfection medium $(1 \mathrm{~mL}$ 
per plate containing cells) and incubated 4 to 5 hours at $37^{\circ} \mathrm{C}$ and low-level $\mathrm{CO}_{2}(3 \%)$. Medium was changed and cells were incubated in growth medium at $37^{\circ} \mathrm{C}, 5 \% \mathrm{CO}_{2}$, for 60 to 72 hours after transfection to generate and release viral particles in the medium. Following this period, the supernatant from plates was collected and cleared of debris by low-speed centrifugation (200 $\mathrm{g}, 5$ minutes), filtered through $0.25-\mu \mathrm{M}$ filters, and spun at 120,000 $\mathrm{g}$ for 1.5 hours (Beckman Coulter Optima ultracentrifuge, rotor SW28), and the pellet was resuspended in PBS.

The titers of viruses were determined by infecting HEK293 cells seeded in 96-well plates at $4 \times 10^{4}$ cells/well the day before infection with serial dilutions of the virus stocks in transfection medium. After overnight incubation, the cell culture medium was changed, and 48 hours later, the number of infected cells and the actual viral titers were scored by counting $\mathrm{EGFP}^{+}$cells across dilutions of viral stock.

hNSC culture. Human H9-derived (WiCell, hPSCreg WAe009-A) hNSCs (Gibco, Thermo Fisher Scientific) were cultured as a monolayer on plasticware (Nunc) coated with CELLstart (Gibco, Thermo Fisher Scientific) in growth medium containing KnockOUT DMEM/F-12 supplemented with $2 \%$ StemPro Neural Supplement, $20 \mathrm{ng} / \mathrm{mL}$ basic FGF (bFGF), $20 \mathrm{ng} / \mathrm{mL}$ EGF, 2 mM GlutaMAX, and 1\% Antibiotic-Antimycotic (cells and culture reagents were obtained from Life Technologies); the medium was changed every 2 days. For plating of hNSCs, $10 \mu \mathrm{M}$ ROCK inhibitor (Selleckchem) was added to the medium, and 24 hours after plating, the medium was changed to growth medium without ROCK inhibitor. When growth was approximately $90 \%$ confluent, cells were split with StemPro Accutase (Life Technologies).

An alternative source of hNSCs was generated from the hESC line RC17 (Roslin Cells, hPSCreg RCe021-A). RC17 hESCs were maintained on plates coated with growth factor-reduced Matrigel (Thermo Fisher Scientific) in StemMACS iPS-Brew XF (Miltenyi Biotec). Cells were passaged with EDTA (0.5 $\mathrm{mM})$ and supplemented with ROCK inhibitor (10 $\mu \mathrm{M}$ Y-27632) on days of passaging. For differentiation into NSCs, RC17 hESCs were seeded at 10,000 cells $/ \mathrm{cm}^{2}$ onto Matrigel-coated plates and cultured for 9 days in N2 medium (1:1 mix of DMEM/F12 and neurobasal with 1\% N2 supplement and $200 \mathrm{mM}$ L-glutamine) supplemented with $10 \mu \mathrm{M}$ SB431542, $100 \mathrm{ng} / \mathrm{mL}$ rhNoggin (i.e., dual SMAD inhibition), and 100 $\mathrm{ng} / \mathrm{mL}$ FGF8b for dorsal forebrain specification. The cells were passaged and cultured from day 9 to day 15 in N2 medium with 1\% B27 supplement, $20 \mathrm{ng} / \mathrm{mL}$ EGF, and $20 \mathrm{ng} / \mathrm{mL}$ bFGF. On day 15, cells were passaged again, and medium was changed to DMEM/F12 with 2\% StemPro neural supplement, $20 \mathrm{ng} /$ $\mathrm{mL}$ EGF, and $20 \mathrm{ng} / \mathrm{mL}$ bFGF. This same medium was used to further expand and maintain the $\mathrm{RC} 17$ NSCs. StemPro Accutase was used for passaging the RC17 NSCs, and $10 \mu \mathrm{M}$ ROCK inhibitor was added to the medium on all days of passaging.

Split transduction of $h N S C s$ and preparation for transplantation. In order to achieve a high efficiency of the viral transduction of hNSCs, transduction was performed while splitting hNSC cultures by using a potentially novel split transduction protocol. The cells were detached with StemPro Accutase and centrifuged ( 5 minutes, $200 \mathrm{~g}$ ); the supernatant with cells was resuspended in $1 \mathrm{~mL}$ of the growth medium; and the cells were counted and then centrifuged again. Supernatant was removed and 20 to $30 \mu \mathrm{L}$ of viral solution in PBS was added to the cell pellet (with multiplicity of infection $=2$ ). Virus and cells were carefully mixed, avoiding bubbles, and an equal amount of growth medium was added to the suspension. Then, cells were incubated at $37^{\circ} \mathrm{C}$ for 10 minutes. To remove debris that could inhibit cell survival, cells were washed in $30 \mathrm{~mL}$ of the growth medium before plating, then centrifuged ( 5 minutes, $200 \mathrm{~g}$ ), resuspended in growth medium, and plated at a density of $0.5 \times 10^{5} \mathrm{cells} / \mathrm{cm}^{2}$ in growth medium containing $10 \mu \mathrm{M}$ ROCK inhibitor. Twenty-four hours after plating, the medium was changed to the growth medium without ROCK inhibitor and growth factors and incubated for 4 days to initiate differentiation of hNSCs. The cells were collected for transplantation by treatment with StemPro Accutase, followed by centrifugation at $200 \mathrm{~g}$ for 5 minutes and washing the pellet twice by resuspension in Dulbecco's PBS (without $\mathrm{Ca}^{2+}$ and $\mathrm{Mg}^{2+}$ ) and centrifuged again. The final dilution of cells was in Leibovitz's L-15 medium (MilliporeSigma) at a concentration of 50,000 cells $/ \mu \mathrm{L}$.

Stereotactic transplantation. Cells were kept on ice for the entire procedure before they were transplanted into the brain. Stereotactic surgery was performed as reported previously $(41,42)$. NSG mice were preanesthetized in an isoflurane chamber and transferred in the stereotactic frame with a gas mask (Angle Two, Leica or Kopf) that had a continuous flow of isoflurane. After applying local anesthesia and making a short skin incision, the skull above the transplantation site was labeled, and a hole was drilled in the skull with a fine syringe needle. The injections were made with glass capillaries using 
Table 1. Primary antibodies used for immunocytochemistry and immunohistochemistry

\begin{tabular}{|c|c|c|c|}
\hline Antibody & Dilution & Catalog & Company \\
\hline Mouse anti- $\beta$-tubulin III (Tuj1) & $1: 500$ & MAB5564 & MilliporeSigma \\
\hline Mouse anti-Bcl-x & $1: 500$ & sc-8392 & Santa Cruz Biotechnology \\
\hline Rabbit anti-cleaved caspase-3 & 1:1000 & 96645 & R\&D Systems \\
\hline Chicken anti-EGFP & 1:1000 & A10262 & Invitrogen, Thermo Fisher Scientific \\
\hline Rabbit anti-EGFP & 1:1000 & CAB4211 & Invitrogen, Thermo Fisher Scientific \\
\hline Rabbit anti-Ki-67 & $1: 500$ & ab15580 & Abcam \\
\hline Goat anti-luciferase & $1: 1000$ & NB100-1677SS & Novus Biologicals \\
\hline Mouse MAP2 antibody & $1: 500$ & 556320 & BD Pharmingen \\
\hline Rabbit anti-nestin & $1: 500$ & ab7659 & Abcam \\
\hline Chicken anti-NeuN & 1:1000 & ABN91 & MilliporeSigma \\
\hline Rabbit anti-Sox1 & $1: 100$ & 41945 & Cell Signaling Technology \\
\hline
\end{tabular}

the following coordinates (from bregma): anteroposterior +0.9 , mediolateral +1.7 , dorsoventral -3.0 (for $\mathrm{H} 9 \mathrm{hNSCs}$ ), and anteroposterior $(\mathrm{A} / \mathrm{P})+0.5$, mediolateral (L/M) +1.7, dorsoventral (D/V) -3.0 (for RC17 hNSCs). Then, $1 \mu \mathrm{L}$ (50,000 cells) was slowly injected into each transplantation site using an oil pressure pump, followed by 3 minutes' incubation of the capillary at the injection site and slow retrieval of the capillary.

C57BL/ 6 mice underwent a similar procedure for transplantation. To reduce innate immune response that could affect survival of human cells in mouse brain, C57BL/ 6 mice were immunosuppressed by daily injections of cyclosporine $(15 \mathrm{mg} / \mathrm{kg} / \mathrm{d})$ and prednisolone $(2 \mathrm{mg} / \mathrm{kg} / \mathrm{d})$ that started 2 days before the transplantations and were performed until sacrificing mice for immunohistochemistry. Note that in spite of immunosuppression, there were a number of transplants (both control and genetically modified) that were rejected by the immune system.

Immunocytochemistry and immunohistochemistry. Immunolabeling was performed as described previously $(43,44)$. Briefly, for immunocytochemical staining, NSC cultures were fixed by cold $4 \%$ paraformaldehyde (PFA, Merck) for 10 minutes, washed with PBS followed by permeabilization with $0.2 \%$ Triton $\mathrm{X}-100$, and blocked with 5\% BSA for 30 minutes. Primary antibodies were incubated overnight in $0.1 \%$ Triton and $5 \%$ BSA at $4{ }^{\circ} \mathrm{C}$, followed by washing with PBS and incubation with secondary antibodies in $0.1 \%$ Triton and $5 \%$ BSA at room temperature.

For immunohistochemistry, mice were deeply anesthetized by injecting ketamine/xylazine intraperitoneally and transcardially perfused, and the brains were postfixed in 4\% PFA overnight. Brain sections (50 $\mu \mathrm{m})$ were prepared using a Leica VT1000S vibratome (Leica). Free-floating sections were permeabilized with $0.2 \%$ Triton $\mathrm{X}-100$ and blocked by $5 \%$ BSA and incubated in $0.1 \%$ Triton and $5 \%$ BSA at $4^{\circ} \mathrm{C}$ with primary antibodies. Sections were washed and secondary antibody incubation was carried out at room temperature for 4 hours. Primary antibodies that were used are presented in Table 1.

As secondary antibodies, we used Alexa Fluor 488-conjugated anti-rabbit (A-11008), anti-mouse (A-11001), anti-chicken (A-11039), and anti-goat (A-11055); Alexa Fluor 546-conjugated anti-mouse (A-11003) and anti-rabbit (A-11035); Alexa Fluor 594-conjugated anti-rabbit (A-21207); and Alexa Fluor 647conjugated anti-mouse (A-31571) and anti-rabbit (A-31573) (1:1000, Invitrogen, Thermo Fisher Scientific). DAPI (MilliporeSigma) was used to label cell nuclei.

Double labeling was quantified confocally; see Supplemental Figure 3 as an example.

$M C A O$. As a stroke model, we induced permanent focal cerebral ischemia. Here, the distal middle cerebral artery (MCA) was occluded by electrical coagulation in 8-week-old NSG mice (45). First, mice 
were anesthetized with tribromoethanol ( $25 \mu \mathrm{L}$ of $1.5 \%$ tribromoethanol/g body weight). Then, the mice were placed under a microscope (Hund), and rectal temperature was maintained at $37^{\circ} \mathrm{C}$ with a heating pad. A skin incision was made between the ear and the orbit on the left side. After removing the temporal muscle, a burr hole was drilled and the meninges were removed. The exposed left MCA was occluded by microbipolar electrocoagulation (ICC 50, Erbe). Finally, the skin incision was sutured and the mice were placed under a heating lamp until they fully recovered. After 48 hours, mice were anesthetized with $1 \%$ to $2 \%$ isoflurane and fixed in a stereotaxic frame (David Kopf Instruments, 1900) to inject hNSCs into the peri-infarct area (the area of injection matched the area of the infarct zone). The following coordinates were used for injecting the cell suspension $(50,000$ cells in $1 \mu \mathrm{L})$ or vehicle (sham): $\mathrm{A} / \mathrm{P}-2.0, \mathrm{~L} / \mathrm{M}+2.3$, $\mathrm{D} / \mathrm{V}-2.5$ (for $\mathrm{H} 9 \mathrm{hNSCs}$ ) and $\mathrm{A} / \mathrm{P}-0.5, \mathrm{~L} / \mathrm{M}+2.3$, D/V -2.0 (for RC17 hNSCs). H9 hNSCs were transplanted closer to the center of the infarct zone, and thus the majority of them were expected to reside in the damaged cortical area that could not be preserved for histology, thus precluding reliable calculations of $\mathrm{H} 9$ hNSC survival in the MCAO model. RC17 hNSCs were transplanted farther away from the center of the infarct zone, and thus their survival could be quantified.

Behavioral tests. To evaluate sensorimotor function, 3 tests were performed 1 day before MCAO surgery, 7 days after hNSC injection. The latency-to-move and the sticky-tape-removal tests have been described previously (25). For the latency-to-move test, mice were placed at the center of a plain board. The time to cross 1 body length $(7 \mathrm{~cm}$ ) was measured. In the sticky-tape-removal test, a small circular adhesive tape (HERMA, 2212, $8 \mathrm{~mm}$ ) was placed on both forepaws 1 after another. Then, the time until the mouse recognized and started to remove the pad as well as the total time to remove it were determined. The handedness of the mice was investigated using the Collins apparatus as described by Collins $(46,47)$. The apparatus is composed of an acrylic glass box that measures $10.2 \times 6 \times 6 \mathrm{~cm}$. On 1 side a $0.9-\mathrm{cm}$ hole was located to allow the mice to grab for food pellets (Dustless Precision Pellets, 14 mg; Plexx) with only 1 paw at a time. After a fasting period of 14 hours, mice were placed in the Collins apparatus, and a total number of 20 grabbing attempts were observed. The Collins score was determined as follows: grabbing attempt $t_{\text {contralateral paw }} /\left(\right.$ grabbing attempt $_{\text {contralateral paw }}+$ grabbing attempt $_{\text {ipsilateral paw }}$ ).

Statistics. We used GraphPad Prism version 7.00 for Mac OS X, GraphPad Software, https://www. graphpad.com, for all statistical analysis. Because there were no differences between control transplantations that were matched with different genetically modified transplants, control transplantations were joined together in 1 group for comparisons. The distribution of data was analyzed by Kolmogorov-Smirnov, d'Agostino, and Shapiro-Wilk tests. Normally distributed data were analyzed using 2-tailed Student's $t$ test (for 2 groups) or 1-way ANOVA test (for more than 2 groups) with Holm-Šídák or Tukey's post hoc tests that included correction for multiple comparisons. Non-normally distributed data were analyzed using the Kruskal-Wallis test with Dunn's post hoc tests. For simultaneous comparison of 2 parameters between 2 or more groups, we used the 2-way ANOVA and Bonferroni's post hoc test. Equality of variances was analyzed using Bartlett's test.

\section{Author contributions}

KK and MS conceptualized and supervised the study. KK, MS, IK, SR, DGG, UP, AK, IS, and AB designed the study, performed experiments, and analyzed data. KK, MS, IK, and SR wrote the manuscript. OD analyzed the infarct zone. All authors edited the manuscript.

\section{Acknowledgments}

We thank Norbert Balint for the help with preparation of brain sections. This study was funded by the Novo Nordisk Foundation's Hallas-Møller Emerging Investigator Grant (NNF16OC0019920) and Lundbeck-NIH BRAIN Initiative (2017-2241) to KK, German Research Foundation funding (SCHW416/5-2) to MS, and Novo Nordisk Foundation grants NNF17CC0027852 and NNF18OC0030286 to AK.

Address correspondence to: Konstantin Khodosevich, Biocenter Research and Innovation Centre, University of Copenhagen, Ole Maaløes Vej, 5. 2200, Copenhagen N, Denmark. Phone: 45.35.33.25.33; E-mail: konstantin.khodosevich@bric.ku.dk. Or to: Markus Schwaninger, Institute for Pharmacology and Toxicology, University of Lübeck, Ratzeburger Allee 160, 23562 Lübeck, Germany. Phone: 49.451.31017200; Email: markus.schwaninger@uni-luebeck.de. 
1. Hermann DM, Peruzzotti-Jametti L, Schlechter J, Bernstock JD, Doeppner TR, Pluchino S. Neural precursor cells in the ischemic brain - integration, cellular crosstalk, and consequences for stroke recovery. Front Cell Neurosci. 2014;8:291.

2. Li JY, Christophersen NS, Hall V, Soulet D, Brundin P. Critical issues of clinical human embryonic stem cell therapy for brain repair. Trends Neurosci. 2008;31(3):146-153.

3. Brederlau A, et al. Transplantation of human embryonic stem cell-derived cells to a rat model of Parkinson's disease: effect of in vitro differentiation on graft survival and teratoma formation. Stem Cells. 2006;24(6):1433-1440.

4. Shear DA, et al. Stem cell survival and functional outcome after traumatic brain injury is dependent on transplant timing and location. Restor Neurol Neurosci. 2011;29(4):215-225.

5. Paul G, Christophersen NS, Raymon H, Kiaer C, Smith R, Brundin P. Tyrosine hydroxylase expression is unstable in a human immortalized mesencephalic cell line--studies in vitro and after intracerebral grafting in vivo. Mol Cell Neurosci. 2007;34(3):390-399.

6. Hicks AU, et al. Transplantation of human embryonic stem cell-derived neural precursor cells and enriched environment after cortical stroke in rats: cell survival and functional recovery. Eur J Neurosci. 2009;29(3):562-574.

7. Ohnishi $\mathrm{K}$, et al. Premature termination of reprogramming in vivo leads to cancer development through altered epigenetic regulation. Cell. 2014;156(4):663-677.

8. Roy NS, Cleren C, Singh SK, Yang L, Beal MF, Goldman SA. Functional engraftment of human ES cell-derived dopaminergic neurons enriched by coculture with telomerase-immortalized midbrain astrocytes. Nat Med. 2006;12(11):1259-1268.

9. Liang Y, Ågren L, Lyczek A, Walczak P, Bulte JW. Neural progenitor cell survival in mouse brain can be improved by co-transplantation of helper cells expressing bFGF under doxycycline control. Exp Neurol. 2013;247:73-79.

10. Gowing G, et al. Glial cell line-derived neurotrophic factor-secreting human neural progenitors show long-term survival, maturation into astrocytes, and no tumor formation following transplantation into the spinal cord of immunocompromised rats. Neuroreport. 2014;25(6):367-372.

11. Hansen C, et al. $\alpha$-Synuclein propagates from mouse brain to grafted dopaminergic neurons and seeds aggregation in cultured human cells. J Clin Invest. 2011;121(2):715-725.

12. Pfisterer U, Khodosevich K. Neuronal survival in the brain: neuron type-specific mechanisms. Cell Death Dis. $2017 ; 8$ (3):e2643.

13. Khodosevich K, Alfonso J, Monyer H. Dynamic changes in the transcriptional profile of subventricular zone-derived postnatally born neuroblasts. Mech Dev. 2013;130(6-8):424-432.

14. Benn SC, Woolf CJ. Adult neuron survival strategies--slamming on the brakes. Nat Rev Neurosci. 2004;5(9):686-700.

15. Kim WR, et al. Impaired migration in the rostral migratory stream but spared olfactory function after the elimination of programmed cell death in Bax knock-out mice. J Neurosci. 2007;27(52):14392-14403.

16. Sinor AD, Lillien L. Akt-1 expression level regulates CNS precursors. J Neurosci. 2004;24(39):8531-8541.

17. Feliciano DM, Zhang S, Quon JL, Bordey A. Hypoxia-inducible factor 1a is a Tsc1-regulated survival factor in newborn neurons in tuberous sclerosis complex. Hum Mol Genet. 2013;22(9):1725-1734.

18. Song MS, Salmena L, Pandolfi PP. The functions and regulation of the PTEN tumour suppressor. Nat Rev Mol Cell Biol. 2012;13(5):283-296.

19. Ahmed NN, Grimes HL, Bellacosa A, Chan TO, Tsichlis PN. Transduction of interleukin-2 antiapoptotic and proliferative signals via Akt protein kinase. Proc Natl Acad Sci USA. 1997;94(8):3627-3632.

20. Khodosevich K, Monyer H. Signaling in migrating neurons: from molecules to networks. Front Neurosci. $2011 ; 5: 28$.

21. Khodosevich $\mathrm{K}$, et al. Connexin 45 modulates the proliferation of transit-amplifying precursor cells in the mouse subventricular zone. Proc Natl Acad Sci USA. 2012;109(49):20107-20112.

22. Khodosevich K, Seeburg PH, Monyer H. Major signaling pathways in migrating neuroblasts. Front Mol Neurosci. 2009;2:7.

23. Bouet V, et al. The adhesive removal test: a sensitive method to assess sensorimotor deficits in mice. Nat Protoc. 2009;4(10):1560-1564.

24. Khan MA, et al. Hyperglycemia in stroke impairs polarization of monocytes/macrophages to a protective noninflammatory cell type. J Neurosci. 2016;36(36):9313-9325.

25. Rahman M, et al. The $\beta$-hydroxybutyrate receptor HCA2 activates a neuroprotective subset of macrophages. Nat Commun. 2014;5:3944.

26. Iadecola C, Anrather J. The immunology of stroke: from mechanisms to translation. Nat Med. 2011;17(7):796-808.

27. Haus DL, et al. Transplantation of human neural stem cells restores cognition in an immunodeficient rodent model of traumatic brain injury. Exp Neurol. 2016;281:1-16.

28. Wu W, Chen X, Hu C, Li J, Yu Z, Cai W. Transplantation of neural stem cells expressing hypoxia-inducible factor-1alpha (HIF-1alpha) improves behavioral recovery in a rat stroke model. J Clin Neurosci. 2010;17(1):92-95.

29. Brunet A, Datta SR, Greenberg ME. Transcription-dependent and -independent control of neuronal survival by the PI3K-Akt signaling pathway. Curr Opin Neurobiol. 2001;11(3):297-305.

30. Navé BT, Ouwens M, Withers DJ, Alessi DR, Shepherd PR. Mammalian target of rapamycin is a direct target for protein kinase B: identification of a convergence point for opposing effects of insulin and amino-acid deficiency on protein translation. Biochem J. 1999;344 Pt 2:427-431.

31. Srinivasan S, Anitha M, Mwangi S, Heuckeroth RO. Enteric neuroblasts require the phosphatidylinositol 3-kinase/Akt/Forkhead pathway for GDNF-stimulated survival. Mol Cell Neurosci. 2005;29(1):107-119.

32. Fuchs C, et al. Loss of CDKL5 impairs survival and dendritic growth of newborn neurons by altering AKT/GSK-3 $\beta$ signaling. Neurobiol Dis. 2014;70:53-68.

33. Androutsellis-Theotokis A, et al. Notch signalling regulates stem cell numbers in vitro and in vivo. Nature. 2006;442(7104):823-826

34. Ka M, Smith AL, Kim WY. MTOR controls genesis and autophagy of GABAergic interneurons during brain development. Autophagy. 2017;13(8):1348-1363.

35. Youle RJ, Strasser A. The BCL-2 protein family: opposing activities that mediate cell death. Nat Rev Mol Cell Biol. 2008;9(1):47-59.

36. Yin W, et al. TAT-mediated delivery of Bcl-xL protein is neuroprotective against neonatal hypoxic-ischemic brain injury via inhibition of caspases and AIF. Neurobiol Dis. 2006;21(2):358-371.

37. Gascón S, et al. Identification and successful negotiation of a metabolic checkpoint in direct neuronal reprogramming. Cell Stem Cell. 2016;18(3):396-409.

38. Savitz SI, Dinsmore JH, Wechsler LR, Rosenbaum DM, Caplan LR. Cell therapy for stroke. NeuroRx. 2004;1(4):406-414. 
39. Beretta S, et al. Effects of human ES-derived neural stem cell transplantation and kindling in a rat model of traumatic brain injury. Cell Transplant. 2017;26(7):1247-1261.

40. Shultz LD, Ishikawa F, Greiner DL. Humanized mice in translational biomedical research. Nat Rev Immunol. 2007;7(2):118-130.

41. García-González D, Khodosevich K, Watanabe Y, Rollenhagen A, Lübke JHR, Monyer H. Serotonergic projections govern postnatal neuroblast migration. Neuron. 2017;94(3):534-549.e9.

42. Khodosevich K, Lazarini F, von Engelhardt J, Kaneko H, Lledo PM, Monyer H. Connective tissue growth factor regulates interneuron survival and information processing in the olfactory bulb. Neuron. 2013;79(6):1136-1151.

43. Khodosevich K, et al. Coexpressed auxiliary subunits exhibit distinct modulatory profiles on AMPA receptor function. Neuron. 2014;83(3):601-615.

44. Watanabe Y, Khodosevich K, Monyer H. Dendrite development regulated by the schizophrenia-associated gene FEZ1 involves the ubiquitin proteasome system. Cell Rep. 2014;7(2):552-564.

45. Lubjuhn J, et al. Functional testing in a mouse stroke model induced by occlusion of the distal middle cerebral artery. $J$ Neurosci Methods. 2009;184(1):95-103.

46. Collins RL. On the inheritance of handedness. I. Laterality in inbred mice. J Hered. 1968;59(1):9-12.

47. Collins RL. On the inheritance of handedness. II. Selection for sinistrality in mice. J Hered. 1969;60(3):117-119. 